\title{
A Multiobjective Optimization for Train Routing at the High-Speed Railway Station Based on Tabu Search Algorithm
}

\author{
Ziyan Feng (iD, Chengxuan Cao $(\mathbb{D}$, Yutong Liu, and Yaling Zhou \\ State Key Laboratory of Rail Traffic Control and Safety, Beijing Jiaotong University, Beijing 100044, China \\ Correspondence should be addressed to Chengxuan Cao; cxcao@bjtu.edu.cn
}

Received 10 June 2018; Accepted 16 September 2018; Published 3 October 2018

Academic Editor: Mahmoud Mesbah

Copyright (C) 2018 Ziyan Feng et al. This is an open access article distributed under the Creative Commons Attribution License, which permits unrestricted use, distribution, and reproduction in any medium, provided the original work is properly cited.

\begin{abstract}
This paper focuses on the train routing problem at a high-speed railway station to improve the railway station capacity and operational efficiency. We first describe a node-based railway network by defining the turnout node and the arrival-departure line node for the mathematical formulation. Both considering potential collisions of trains and convenience for passengers' transfer in the station, the train routing problem at a high-speed railway station is formulated as a multiobjective mixed integer nonlinear programming model, which aims to minimize trains' departure time deviations and total occupation time of all tracks and keep the most balanced utilization of arrival-departure lines. Since massive decision variables for the large-scale real-life train routing problem exist, a fast heuristic algorithm is proposed based on the tabu search to solve it. Two sets of numerical experiments are implemented to demonstrate the rationality and effectiveness of proposed method: the small-scale case confirms the accuracy of the algorithm; the resulting heuristic proved able to obtain excellent solution quality within 254 seconds of computing time on a standard personal computer for the large-scale station involving up to 17 arrival-departure lines and 46 trains.
\end{abstract}

\section{Introduction}

Generally, the station is composed of bottleneck and arrivaldeparture lines which is vital for trains arriving, departing, running, shunting operations, and so on. The efficiency of railway transport faces challenges due to increasing passengers and freight transportation demands. In order to enhance the capacity of the railway station, operators can be dedicated to adding the number of track lines, which requires plenty of manpower, materials, and financial resources obviously. By contrast, making proper arrangements of trains' routes at bottleneck and arrival-departure lines based on existing track lines can save lots of resources and achieve the same effect. Hence, attention is paid to arrange the trains' routes reasonably in the station to improve the operational efficiency and reduce the operation costs [1,2] (D’Ariano et al., 2008).

In general, the railway planning process is divided into strategic level, tactic level, and operational level. In this paper, we focus on the tactic level, especially on the timetable optimization and railway track allocation/train routing in a complex high-speed railway station. At present, the optimization of the train routing problem (TRP) in the railway station is mainly based on the mathematical optimization model. Some studies aimed at the complicated bottleneck of the railway station, as D'Ariano et al. [3] considered that reducing trains' collisions at the bottleneck can improve punctuality without decreasing the capacity usage of the lines and a detailed model for conflict resolution and different algorithms was illustrated. Based on the robustness of routing assignment, Jia et al. [4] proposed the optimization of routing utilization at bottleneck. Kang et al. [2] presented a bottleneck optimization model thereafter to enhance the capacity by reasonably arranging routes and turnouts. Other parts of the researches concentrated on the problems of arrival-departure lines occupancy. For example, Billionnet [5] considered assigning trains to the available tracks at a railway station instead of taking into account the collision at the bottleneck. Caprara et al. [1] proposed a 0-1 integer programming model to describe the routing problem of trains at the station platform; moreover, the quadratic objective function was linearized and solved by integer linear programming. Besides, an optimization model is presented by Qiao et al. [6] based on the train schedules to arrange suitable arrival-departure lines for passenger trains. Furthermore, the research of the TRP 
with joint optimization of bottleneck and arrival-departure lines is also found in the literature. For instance, Zwaneveld et al. [7] considered both bottleneck and arrival-departure lines and proposed a $0-1$ programming model to arrange trains passing the railway station. But the problem only at smallscale railway stations can be solved by branch-cut method. Based on the graph theory, Corman et al. [8] rearranged train routing in real-time unpredictable events and found the best solution using truncated branch-and-bound and tabu search algorithms.

In the past few decades, there have been limited researches on TRP in high-speed railway stations. Most studies were associated with the train timetable problems [911]. TRP is tantamount to selecting a sequence of tracks for a train from its origin to destination, with the objective of minimizing the sum of travel time, the total operating cost, and/or increasing the capacity of railway network. Xu et al. [11] defined the objective function to minimize deviations between trains' arrival time at the destination and original timetable. The optimization model proposed by $\mathrm{Li}$ et al. [12] aimed to minimize the total delay of all trains in the railway network. Liu et al. [13] developed a mathematical model whose aim is minimizing the total occupation time of station bottleneck sections to avoid train delays. In addition, some research focused on the optimization by keeping the most balanced utilization arrival-departure lines like Qiao et al. [6]. One of the objective functions considered by Zhou et al. [14] and Zhou et al. [10] was to minimize the total travel time on the track. Apart from this, the most important constraint should be taken into account was the spatiotemporal interactions between each train operation route in the TRP (D'Ariano et al., 2008) [2, 4, 12]. Furthermore, it should be considered that trains which occupy the same arrival-departure lines should satisfy a headway time constraint [1, 6]. Zhou et al. [14] and Corman et al. [8] proposed that trains should stop for enough time to ensure the transfer time of passengers and crews. What is more, Fang et al. [15] analyzed a comprehensive survey on different models by a clear classification based on the different scale, infrastructures, objectives, and constraints.

It is well known that the TRP is the NP-hard problem [16] and unlikely to get an exact optimal solution in a short computational time under the large-scale and complex situation. In order to get an approximate optimal solution rapidly, many studies proposed different heuristic algorithms based on different strategies. Corman and Meng [17] introduced the online dynamic models and algorithms for the rail traffic management in order to provide punctual and reliable services. Specifically, Ahuja et al. [18] considered the issue of railway scheduling and presented a heuristic algorithm to get the approximate optimal solution in short time. Carey et al. [19] studied the large-scale problem that involved lots of train schedules and routes and proposed heuristic algorithms to solve it. Liu et al. [13] designed a hybrid algorithm between genetic algorithm and the simulated annealing algorithm. In recent years, for the sake of further improving the computational speed and quality of solution, some studies have proposed improving heuristic algorithms. He et al. [20] proposed an improve branch-and-price algorithm to deal with the large scale integer programming. Zhou et al. [10] used an efficient train-based lagrangian relaxation decomposition to the simultaneous passenger train routing and timetabling problem. Additionally, some studies used commercial software like Qi et al. [21] who obtained for an approximate optimal solution within 30 s by a local search heuristic algorithm using CPLEX solver and Xu et al. [11] who solved train routing and timetabling problem with switchable rules by CPLEX solver with OPL language.

Besides that, some studies dealt with the integer programming problem using tabu search algorithm, as Isaai and Singh combined the heuristic with tabu search and simulated annealing search control strategies to deal with the train timetabling problem. Similar, Corman et al. [8] rearranged trains' routes based on the graph theory and got the approximate optimal solution of the combination of truncated branch-and-bound and tabu search algorithms. Li et al. [12] proposed a tabu search algorithm, and they pointed out that the algorithm relies on a better initial solution; otherwise the result obtained is not stable. In addition to this, Goh et al. proposed a tabu search with sampling and perturbation to generate feasible solutions. The tabu search algorithms and variable neighborhood are applied by Samà et al. [22] to improve the solution for the real-time management problem of scheduling and routing trains in complex and busy railway networks.

As can be found in numerous studies, there were few elaborate mathematical models to describe TRP and further study on TRP in the network of the high-speed railway station. In this research, with the motivation of greatly improving the solving efficiency of TRP in the network of the high-speed railway station, we intend to provide the following contributions:

(i) Describe a railway network by defining the turnout node and the arrival-departure line node on account of the traditional layout of the railway station, which is regarded as a directed graph. The nodes are regarded as vertices, and the actual connection of the lines is regarded as arcs. The railway station is divided into three parts and the connection sets are built to satisfy the connection relationship of them.

(ii) According to the given nominal timetable, the calculation methods of trains occupying each track are elaborated. Then formulate the TRP in the network of the high-speed railway station as a multiobjective mixed integer nonlinear programming model, in which both are considered the potential collisions of trains and the convenience for passengers' transfer in the high-speed railway station. In the proposed model, it is not only minimizing train departure time deviations with the most balanced utilization of arrival-departure lines but also minimizing the total occupation times of all tracks.

(iii) The TRP is NP-hard problem. Therefore, it is very unlikely to devise a polynomial-time (exact) algorithm for it. In order to get an approximate optimal solution rapidly, a heuristic algorithm is proposed 
based on the tabu search to solve the large-scale TRP in the network of the high-speed railway station.

(iv) Numerical examples are implemented to demonstrate the effectiveness and efficiency of proposed method. By taking advantage of an efficient tabu search algorithm, we can solve the model rapidly for a small case. The results we obtained from the algorithm are the same as obtained directly from CPLEX solver. In the large-scale case involving 17 arrival-departure lines and 46 trains between 16:00 19:00, the resulting heuristic proved able to obtain excellent solution quality within 254 seconds of computing time on a standard personal computer.

The remainder of this paper is organized as follows. Section 2 presents a detailed description of TRP problem. Section 3 provides the mathematical formulation for the TRP in the network of the high-speed railway station. Section 4 deals with the development of a heuristic algorithm based on the tabu search. Section 5 describes the instances used and provides computational results. Finally, some conclusions and further research directions are presented in Section 6.

\section{Train Routing Problem Description}

2.1. Layout of Railway Station. We consider a railway station as illustrated in Figure 1. This railway station consists of bottlenecks and some arrival-departure lines whose length is $L_{s}$. The left side of the station in Figure 1 is defined as left bottleneck whose length is $L_{l}$. The route set of it is $L=\left\{l_{1}, l_{2}, \cdots, l_{k_{1}}\right\}$. Similarly, the right side is defined as right bottleneck whose length is $L_{r}$. The route set of it is $R=\left\{r_{1}, r_{2}, \cdots, r_{k_{2}}\right\}$. The bottleneck involves tracks where trains arrive and depart. For instance, an outbound train arrives from node $d_{1}$ and stops at arrival-departure line. In Figure 2(a), there are 4 possible routes for the outbound train that arrives from node $d_{1}$, whose set is $L_{1}=\left\{l_{1}, l_{2}, l_{3}, l_{4}\right\}$ in the left bottleneck. Similar, an inbound train departs from arrival-departure line to the node $d_{2}$. There are also 4 possible routes for the inbound train departs from node $d_{2}$ as shown in Figure 2(b) whose set is $L_{2}=\left\{l_{5}, l_{6}, l_{7}, l_{8}\right\}$. So the route set of the left bottleneck is $L=L_{1} \cup L_{2}=\left\{l_{1}, l_{2}, \ldots, l_{8}\right\}$. In particular, at large stations, the number of routes in the bottleneck may be larger than the number of arrival-departure lines due to the existence of multiple crossovers and turnouts. The route set of arrival-departure lines is $S=\left\{s_{1}, s_{2}, \ldots, s_{m}\right\}$, which contains $I, I I, 3$, and 4 as shown in Figure 1 .

Normally, track lines are divided by insulation joints. In this paper, the turnout node and the arrival-departure line node are defined as follows to describe the railway station.

1. Turnout node: the intersection of the lines in the station. It includes turnouts (as shown in Figure 1, $d_{5}$ and $d_{6}$, etc.), crossovers (as shown in Figure $1, d_{7}$, etc.), and the position of signal which located in the entrance of the bottleneck (i.e., the boundary point of the station).

2. Arrival-departure line node: the connection points of bottleneck and arrival-departure lines in the railway station, namely, the signal position of the entrance of the arrival-departure lines (i.e.. the nodes $d_{8} \sim d_{10}$ in Figure 1).

During the operation, the outbound trains can only arrive from turnout node $d_{1}$ and depart from turnout node $d_{3}$ as shown in Figure 1. Similar, the inbound trains can only arrive from turnout node $d_{4}$ and depart from turnout node $d_{2}$. However, no matter in which direction trains are, any of arrival-departure lines can be occupied. Hence the physical network of the railway is regarded as a directed graph. The nodes are regarded as vertices, and the actual connection of the lines is regarded as arcs. Let $D$ denote the set of all vertices and $A$ denote the set of all arcs in the network.

2.2. Trains. We consider a set of trains in both directions. The set of trains is denoted by $C=\left\{c_{1}, c_{2}, \cdots, c_{n}\right\}$, in which $C_{1}=\left\{c_{1}, c_{3}, c_{5}, \cdots\right\}$ is the set of outbound trains and $C_{2}=$ $\left\{c_{2}, c_{4}, c_{6}, \cdots\right\}$ is the set of inbound trains. The heterogeneous trains are taken into account. We assume that these trains are categorized into two types: slow trains and fast trains. In addition, there are shunting operation beside train reception and departure operations. The speeds of shunting trains are slower than the ordinary trains.

In addition, some of the fast trains pass the station without stopping while some trains stop at platform for passengers to alight or transfer. Different trains' stop time may be different, whether they are fast or slow trains. As we all know, the speed of trains is not a constant, while it changes with the driver's braking when the train would stop at the platform. Similarly, the speed of trains does not increase suddenly when departing from the platform. Therefore, the additional time that provides trains' acceleration and deceleration is considered. At the same time, the length of trains is also taken into account to meet the actual situation.

2.3. Conflicts and Potential Conflicts. The conflicts of tracks which trains occupy are the most imperative problem to be solved in TRP. In other words, trains cannot occupy the same track at the same time. A conflict occurs whenever trains traverse the same track and do not respect the minimum time interval at the bottleneck and arrival-departure lines. What is more, it would result in potential conflicts. These situations are discussed separately as follows.

2.3.1. Train Routing Conflicts at Bottleneck. The bottleneck consists of channels with some turnouts, crossovers, and other facilities, which may cause spatial intersections of trains' alternative sets of routes, especially when some unpredictable events or interruptions occurred. So it is an important task for train dispatchers to avoid the train collision and to assure the trains passing the tracks orderly. The conflicts between two trains in the bottleneck mainly occurred at the intersection of the track segments (the segment $d_{1} \sim d_{2}$ as shown in Figure 3 ) and the intersection of crossovers (the point $d_{3}$ as shown in Figure 3 ).

In Figures 3(a) and 3(b), trains $c_{1} / c_{2}$ travel in the same/reverse direction, and the collision occurs at the track segment between nodes $d_{1}$ and $d_{2}$. Similarly, the collision 


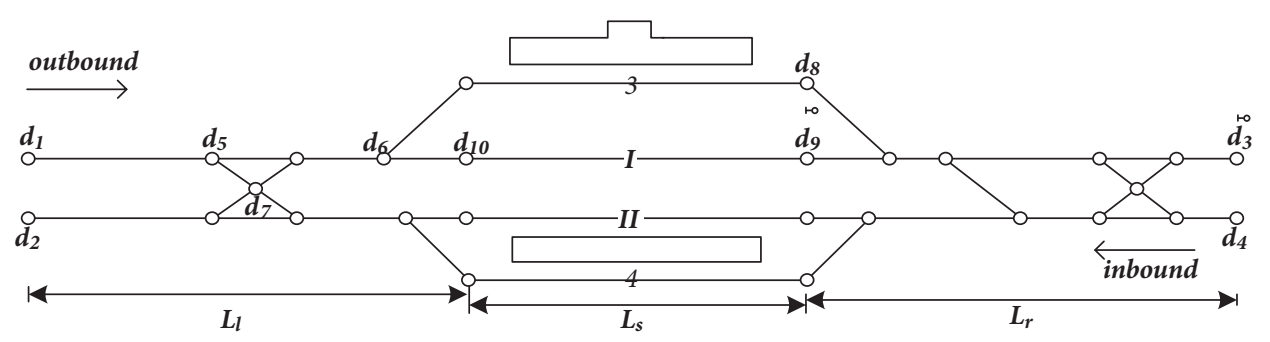

FIGURE 1: An illustration of a railway station.

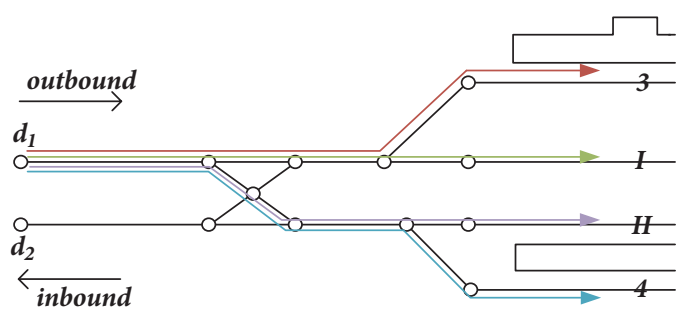

(a)

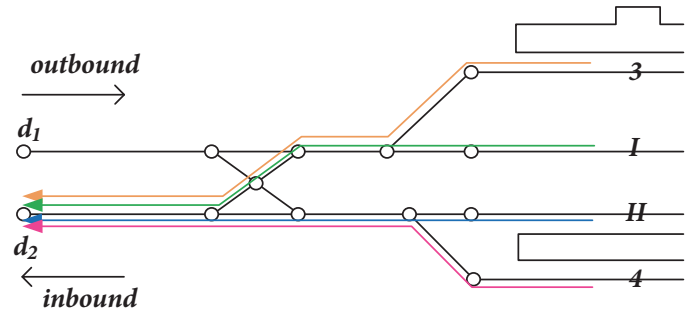

(b)

FIGURE 2: The route set of left bottleneck in the railway station.

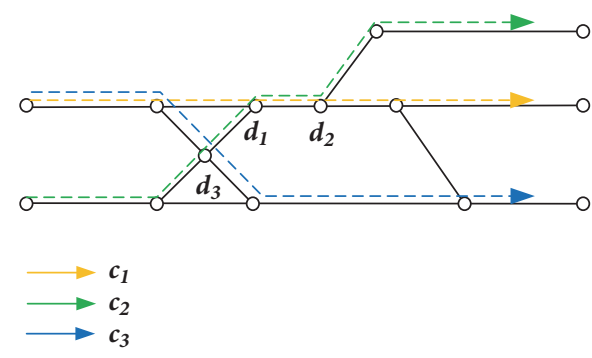

(a)

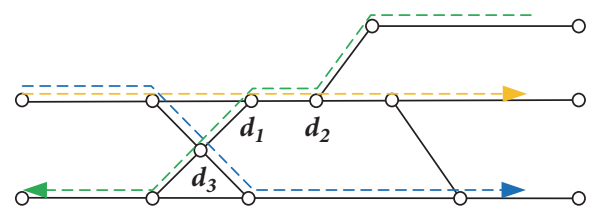

(b)

FIGURE 3: The same/reverse direction conflicts of trains routing.

occurs at the track segment at the node $d_{3}$ when the trains $c_{2}$ and $c_{3}$ travel in the same and reverse direction, respectively. The methods to dissipate conflicts will be depicted in detail in Section 3.

\subsubsection{Train Routing Conflicts at Arrival-Departure Lines.} When two consecutive trains plan to occupy the same arrivaldeparture line in the railway station, they should follow a headway time to suit the limited infrastructure capacity. That is, one train occupies this arrival-departure line a specific headway time later than the other one. As shown in Figure 4, there are two trains $c_{1}$ and $c_{2}$. The origin and destination of train $c_{1}$ are $\mathrm{A}$ and $\mathrm{C}$, respectively, and the origin and destination of train $c_{2}$ are $\mathrm{B}$ and $\mathrm{C}$. Obviously, if the route $p_{1}$ is assigned to $c_{1}$ and $c_{2}$ occupies route $p_{2}$ in a short interval, this would cause a conflict at platform 1 . In this case, the latter train should stop and wait until the previous train leaves from the platform 1 for a reasonable headway time. It may cause the latter train to be behind the schedule time and even to have second delay in severe cases. But if the train $c_{2}$ occupies route $p_{3}$, the conflicts would be avoided and the train operational efficiency would be ensured in the railway station.

Therefore, if the two trains' departure interval is smaller than the headway time $T_{m}^{1}$, it is better to arrange them at different lines. Just as illustrated in Figure 4, if the difference of two trains' departure time is bigger than the headway time $T_{m}^{1}$, the same arrival-departure line can be arranged.

2.3.3. Potential Conflicts of Train Routing. It is noteworthy that only considering trains' routing at bottleneck and headway time at arrival-departure lines cannot completely meet the requirement of safe operation. There are further potential conflicts.

For instance, the outbound train $c_{1}$ would stop at arrivaldeparture line $I I$ from outbound main line while the inbound train $c_{2}$ would depart from line 3 to the inbound main line (as shown in Figure 5(a), the yellow dotted line and the blue dotted line represent the route of trains $c_{1}$ and $c_{2}$, respectively). There is no time provided to avoid the potential conflict area (the red circle area in Figure 5), since it is not in 


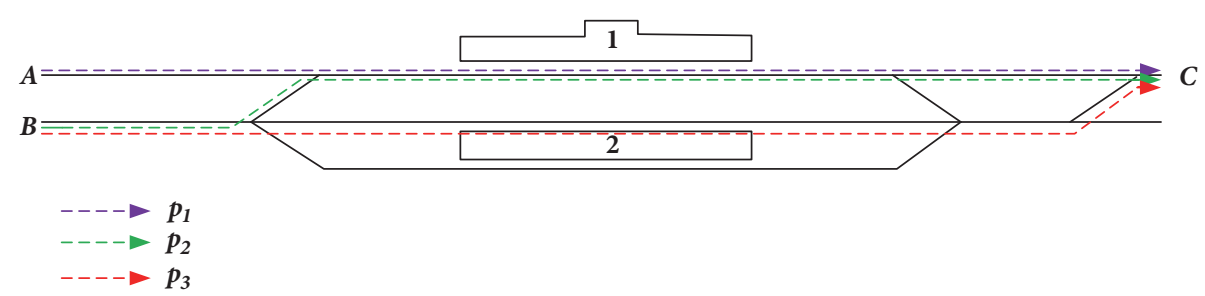

FIgURE 4: The conflict at arrival-departure lines.

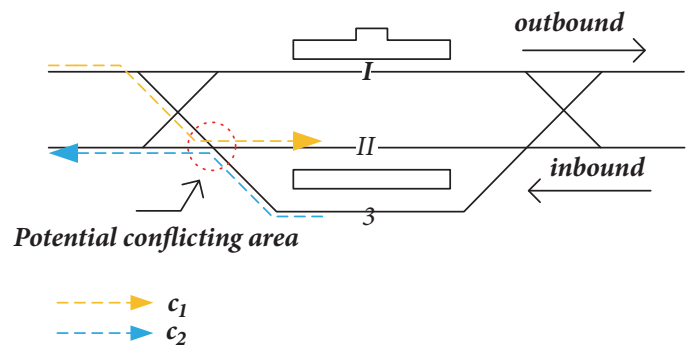

(a)

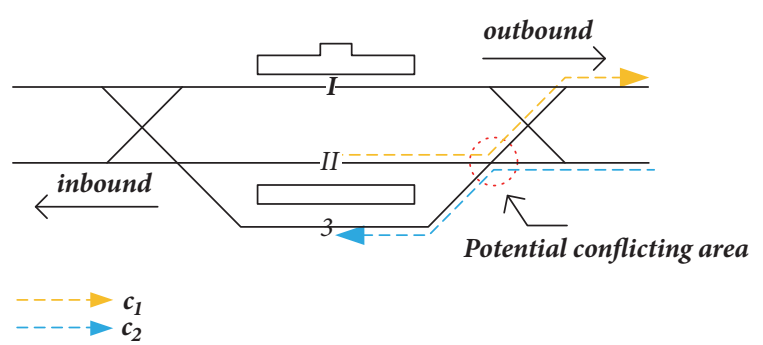

(b)

Figure 5: The illustrative of outbound and inbound trains' potential conflicts.

the context of the two conflicts described above. Therefore, a time separation should be adopted even though two trains do not occupy the same track; i.e., $c_{1}$ first arrives and then $c_{2}$ departs later or $c_{2}$ first leaves and then $c_{1}$ arrives later, the same as shown in Figure 5(b). It is necessary to set constraints so that two consecutive trains arrive the potential conflict area in a time separation.

In addition, when two trains are in the same direction, the conflicts between them are not potential conflicts. Just as shown in Figure 6, $c_{1}$ and $c_{2}$ are outbound trains. It is apparent that there exists a conflict in the red circle area. However, due to the conflicts at the bottleneck between the two trains, the constraints are set to avoid the collision; namely, conflicts in the rad circle area are also dissolved. Therefore, the potential constraints we discussed are only among the reverse trains.

2.4. The Calculation Methods of Track Occupation Time. In this paper, not only is the train routing chosen as the decision variables but also optimized the schedule of trains to start entering the station according to the planned schedule when trains depart from the station. Thus the time for trains passing the conflict tracks needs be calculated. In addition, the convenience factors of passengers transferring at station are taken into account, and the associated trains' dwell-time intersection should be long enough. As a consequence, the trains' travel time in different track sections should be clearly calculated.

To our knowledge, as long as a track segment is occupied, the lines, the fouling posts, and the signals are also occupied at the same time. We elaborate the calculation of the occupied time and the end time of occupied of trains passing through the left bottleneck, the arrival-departure lines, and the right bottleneck in turn. The occupied trains at the bottleneck are divided into two situations: train reception and train departure, whose calculated method of train occupied time of tracks is same. So only take the outbound trains as an example to explain in detail.

2.4.1. The Calculation of Trains Travel Time and the End Time of Occupied at the Bottleneck. For outbound trains $c \in C_{1}$ in the left bottleneck $l \in L$, they start to enter the station at $t_{c}+$ $t_{c}^{\text {setup }}$ and pass each node with speed $v_{c}$. The track is unlocked after their tails pass the end node of the track section. So the trains' occupied time of each track segment is calculated as follows:

$$
t_{a}=\frac{L_{a}}{v_{c}}
$$

where $t_{a}$ is occupancy time of a certain track segment $a$ and $L_{a}$ is length of the track segment $a$. Thus, the travel time of outbound trains $c \in C_{1}$ from the left bottleneck signals to the arrival-departure lines is calculated with the following:

$$
t_{c s}^{l}=\frac{\left(L_{l}+L_{c}\right)}{v_{c}}
$$

where $L_{l}$ is length of routes which is sum of each track segment in the left bottleneck and $L_{c}$ is the length of the train $c$. In Figure 7, the length of $L_{d_{1} \sim d_{10}}$ is the sum of lengths of $d_{1} \sim d_{6}$ and $d_{6} \sim d_{10}$; the length of $L_{d_{1}} \sim d_{11}$ is the sum of lengths of $d_{1} \sim d_{6}$ and $d_{6} \sim d_{11}$. That is, the length of the different routes in the left bottleneck is different. The end of the left bottleneck occupied time of outbound trains is

$$
t_{c l}^{\text {end }}=t_{c}+t_{c}^{\text {setup }}+t_{c s}^{l}
$$

Take the railway station in Figure 1 as an example to calculate the occupancy time of one route of outbound train 


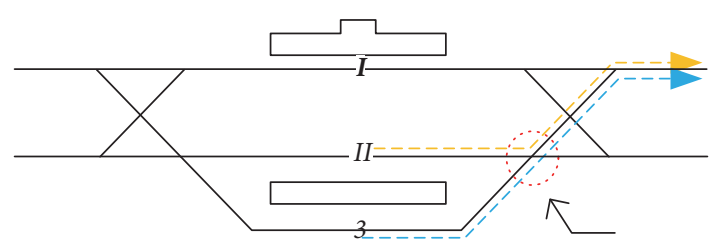

Non-potential conflicting area

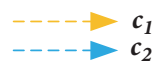

FIGURE 6: The illustrative of the same direction trains occupied different lines.

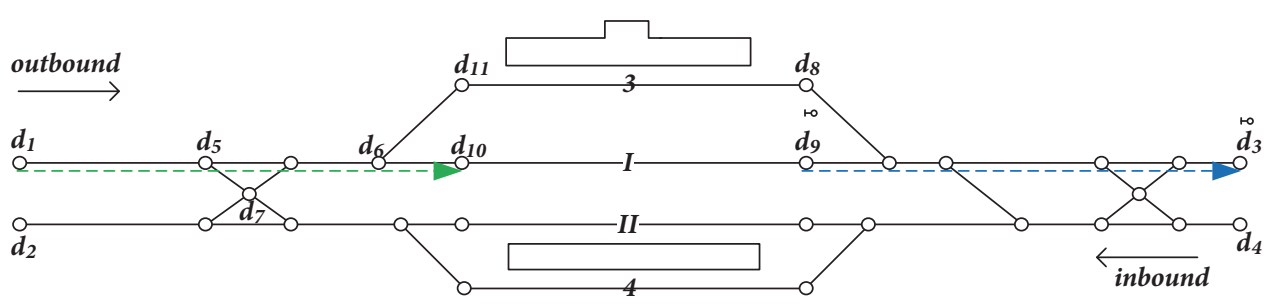

FIGURE 7: The calculation of trains' travel time in station.

in the left bottleneck. As shown in Figure 7, it is assumed that the train arrives from the node $d_{1}$ and stops at line $I$ (i.e., the green dashed line in Figure 7). Then the travel time of train $c$ from the left bottleneck signal to the line $I$ is calculated as $t_{c s_{I}}^{l_{1}}=\left(L_{d_{1} \sim d_{10}}+L_{c}\right) / v_{c}$. The end of the left bottleneck occupied time is $t_{c l_{1}}^{\text {end }}=t_{c}+t_{c}^{\text {setup }}+t_{c s_{I}}^{l_{1}}$.

The occupied time of outbound trains departing from the station through the right bottleneck is calculated as

$$
t_{c s}^{r e}=\frac{\left(L_{r}+L_{c}\right)}{v_{c}}
$$

Similarly, the travel time of trains departing from the line $I$ and leaving to node $d_{3}$ through the right bottleneck (i.e., the blue dashed line in Figure 7) is calculated as $t_{c s_{I}}^{r_{1} e}=\left(L_{d_{9} \sim d_{3}}+\right.$ $\left.L_{c}\right) / v_{c}$.

For the inbound trains $c \in C_{2}$, the calculation of trains travel time and the end time of occupied at right bottleneck are as follows, respectively:

$$
\begin{aligned}
t_{c s}^{r} & =\frac{\left(L_{r}+L_{c}\right)}{v_{c}} . \\
t_{c r}^{\text {end }} & =t_{c}+t_{c}^{\text {setup }}+t_{c s}^{r} .
\end{aligned}
$$

The travel time in the left bottleneck when the train departs from the station is calculated by

$$
t_{c s}^{l e}=\frac{\left(L_{l}+L_{c}\right)}{v_{c}} \text {. }
$$

2.4.2. The Calculation of Trains Travel Time and the End of Occupied Time at Arrival-Departure Lines. The occupation of arrival-departure lines can be partitioned into two kinds. One is that some fast trains pass the station directly without stopping at the platform, and the other one is that trains stop for a time interval before leaving the station.
In the first case, the train's travel time through arrivaldeparture lines can be obtained directly according to the length of the arrival-departure lines and the speed of the trains: $t_{c s}=\left(L_{s}+L_{c}\right) / v_{c}$.

In the second case, we consider the deceleration time $t_{c s}^{d}$ when the speed of train $c$ decelerates to 0 to stop at the platform and the acceleration time $t_{c s}^{a}$ when the train's speed increases to $v_{c}$ from 0 to leave the station. Then the travel time on the arrival-departure lines is $t_{c s}=t_{c s}^{d}+t_{c s}^{a}+t_{c s}^{s}$.

For better expression in both cases, we adopt a binary variable $\tau_{c s}^{s}$ to imply if train $c$ would stop at arrival-departure line $s$. Then the travel time on the arrival-departure lines can be calculated as follows:

$$
t_{c s}=\tau_{c s}^{s}\left(t_{c s}^{d}+t_{c s}^{a}+t_{c s}^{s}\right)+\frac{\left(1-\tau_{c s}^{s}\right)\left(L_{s}+L_{c}\right)}{v_{c}} .
$$

The departure time from the arrival-departure line $s$ of the stopped trains is:

for the outbound trains $c \in C_{1}: t_{c}^{f}=t_{c l}^{e n d}+t_{c s}^{d}+t_{c s}^{s}$,

for the inbound trains $c \in C_{2}: t_{c}^{f}=t_{c r}^{e n d}+t_{c s}^{d}+t_{c s}^{s}$.

The end of occupied time at the arrival-departure lines is given at the same time:

$$
\begin{aligned}
& \text { for the outbound trains } c \in C_{1}: t_{c}^{\text {fend }}=t_{c l}^{\text {end }}+t_{c s} \text {, } \\
& \text { for the inbound trains } c \in C_{2}: t_{c}^{\text {fend }}=t_{c r}^{\text {end }}+t_{c s} \text {. }
\end{aligned}
$$

In general, the relationship of train's occupied time on the track can be illustrated clearly in Figure 8. Figures 8(a) and $8(\mathrm{~b})$ indicate the process of outbound trains and inbound trains passing through the station, respectively. The trends of curves in two graphs are similar since the principle of outbound trains and inbound trains is similar. The ordinate 


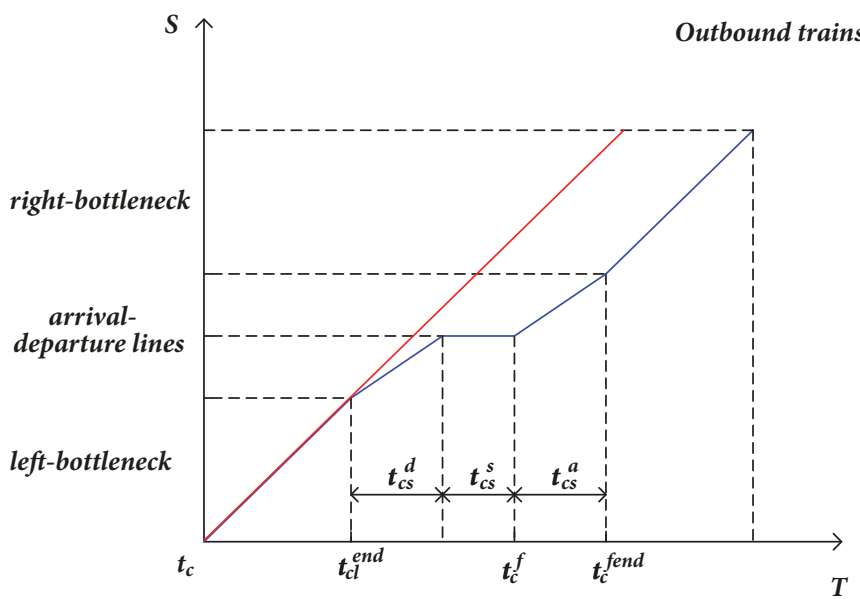

(a)

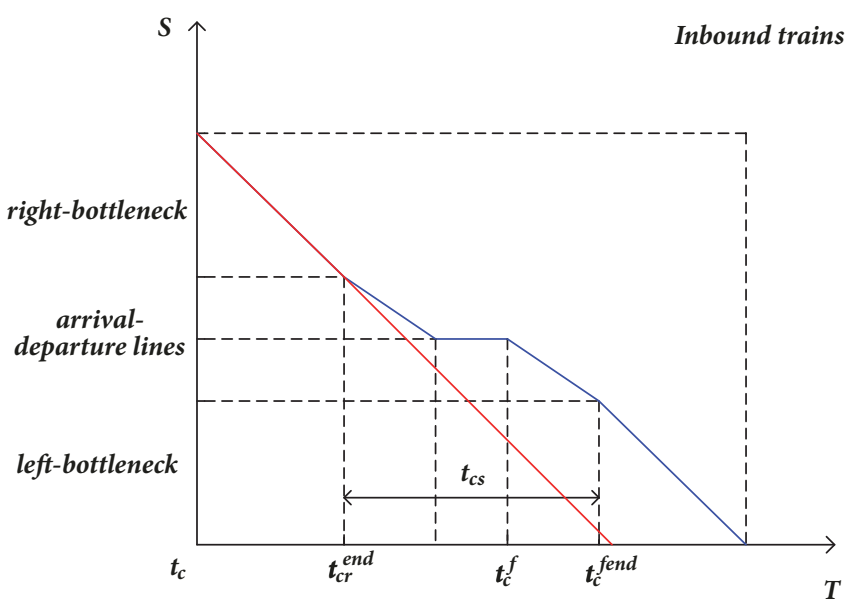

(b)

FIGURE 8: A space-time network of outbound and inbound trains passing through the rail station.

indicates train's position when passing through the station, and the abscissa shows the time correspondingly. The red line represents trains that do not stop at the station and the blue one represents trains that would stop at the station.

\subsection{Problem Statement and Notation}

2.5.1. Problem Description and Assumption. TRP is regarded as a pivotal component in providing high efficiency of operation, which can greatly affect the quality of train schedules and passengers service levels. In practice, TRP is often based on train planning problem, often predesigned by predicted demands and service capability. As mentioned above, the description of the physical railway station is restated in this paper. Moreover, the character of trains, the conflicts which may occur, and the calculation of trains' travel time are introduced. In general, the problems we studied are the train routing optimization problems which involve lots of heterogeneous trains and many safety constraints in a complex high-speed railway station.

Mathematically, we use binary decision variables to represent whether a train is allocated on a track segment or not. When a set of feasible decision variables are determined, the routing for each train can be specified concurrently. Then an integer decision variable is quoted to ensure punctuality of trains based on planned timetable. One of the objectives discussed in this paper is to minimize trains' total travel time on the tracks in the station to ensure trains passing the station in a short time. It reduces the energy consumption and ensures the punctuality rate. It is worth noting that the route is shortest if train is allotted on the sidings close to main lines. Otherwise, the distance would increase if the train is arranged to stop on others, especially the furthest arrival-departure line from the main line. Nevertheless, it would cause serious wear and tear on the tracks if one arrivaldeparture line is often occupied repeatedly. Therefore, it is of importance to maintain the occupancy balance of the arrivaldeparture lines, which is reflected in the number of trains on the arrival-departure lines and the duration time. Finally, the punctuality of trains is considered so that it would not create trains delay to reduce the operation efficiency.

Therefore, a mixed integer programming model is built based on the constraints of safety and station ability. The details and algorithm are discussed in Section 3. The assumptions throughout this paper are listed as follows.

Assumption 1. The trains pass the station at a constant speed $v_{c}$ if they do not stop at the platform. For stopped trains, the additional acceleration and deceleration time of heterogeneous trains are same, respectively, for simplification.

Assumption 2. Due to the safety requirements of the fouling post, trains must stay within the fouling post. So we assume that all trains satisfy the safety requirements of the fouling post to ensure that the tails of trains would not collide.

Assumption 3. Each train has a planned departure time and cannot depart from the station earlier than the predetermined departure time.

2.5.2. Notations. Regarding the trains, input data includes set/index, velocity, length, and other property parameters. In addition, it also covers relationship between trains, the time of trains' setup, travel, and dwelling. Based on this basic input data of trains, we can further determine the relationship between trains and routes and trains occupation time and occupation end time, also predetermined as inputs of our model. As for the railway network, its input data involves the set/index of nodes and arcs, the set of connection of track segments, the length of different areas, and safety time interval and headway time. The details are summarized in Table 1 .

The outputs of TRP compose the traverse route sets of trains when they arrive at and depart form the station and the starting time of trains to pull into the station, as well as their precedence relation of two trains at the same track. The details are introduced in Table 2. 
TABLE 1: The indices, parameters, and sets.

\begin{tabular}{|c|c|}
\hline Notations & Description \\
\hline$C$ & Set of trains \\
\hline$C_{1}, C_{2}$ & Set of outbound trains and inbound trains respectively \\
\hline$c$ & Index of trains \\
\hline$S$ & Set of arrival-departure lines \\
\hline$s$ & Index of arrival-departure lines \\
\hline$L, R$ & Set of routes at left bottleneck and right bottleneck respectively \\
\hline$l, r$ & Index of routes at left bottleneck and right bottleneck respectively \\
\hline$P$ & Set of trains' routes \\
\hline$D, A$ & Set of nodes and arcs of railway network respectively \\
\hline$R_{s}^{l}, R_{s}^{r}$ & Set of left and right bottleneck routes connected to the arrival-departure line $s$ respectively \\
\hline$\alpha_{c}$ & Weight of the heterogeneous trains \\
\hline$\beta$ & Weight of different objective functions \\
\hline$L_{l}, L_{r}$ & Length of routes in the left and the right bottleneck respectively \\
\hline$L_{s}$ & Length of the arrival-departure lines \\
\hline$L_{c}$ & Length of trains \\
\hline$L_{d_{1} d_{2}}$ & Length of the track segment between $d_{1}$ and $d_{2}$ \\
\hline$t_{c}^{*}$ & Planned starting time of train $c$ to pull into the station \\
\hline$t_{c}^{f}$ & Actual departure time of train $c$ from the station \\
\hline$v_{c}$ & Speed of train $c$ \\
\hline$t_{c}^{\text {setup }}$ & Setup time of train $c$ \\
\hline$t_{c l}^{\text {end }}$ & Occupation end time at the left bottleneck of outbound trains \\
\hline$t_{c r}^{\text {end }}$ & Occupation end time at the right bottleneck of inbound trains \\
\hline$t_{c}^{\text {fend }}$ & Occupation end time at the arrival-departure lines \\
\hline$t_{c s}$ & Travel time at the arrival-departure line $s$ of train $c$ \\
\hline$t_{c s}^{s}$ & Dwell time at the arrival-departure line $s$ of train $c$ \\
\hline$t_{c s}^{d}, t_{c s}^{a}$ & Additional times corresponding to acceleration and deceleration of trains, respectively \\
\hline$t_{c s}^{l}, t_{c s}^{r e}$ & Travel time at left bottleneck to arrive and at right bottleneck to depart of outbound train $c$ \\
\hline$t_{c s}^{r}, t_{c s}^{l e}$ & Travel time at right bottleneck to arrive and at left bottleneck to depart of inbound $\operatorname{train} c$ \\
\hline$t_{c_{1} c_{2}}^{c o n}$ & Time interval between trains $c_{1}$ and $c_{2}$ entering the conflict track segments \\
\hline$\tau_{c s}^{s}$ & Binary parameters, if train $c$ would stop at arrival-departure line $s, \tau_{c s}^{s}=1$, else, $\tau_{c s}^{s}=0$ \\
\hline$\gamma_{c_{1} c_{2}}$ & Binary parameters, if potential conflicts exist, $\gamma_{c_{1} c_{2}}=1$, else, $\gamma_{c_{1} c_{2}}=0$ \\
\hline$\theta_{p_{1} p_{2}}$ & Binary parameters, if there are conflicts between routes $p_{1}$ and $p_{2}, \theta_{p_{1} p_{2}}=1$, else, $\theta_{p_{1} p_{2}}=0, l, s, r \in p$ \\
\hline$\delta_{s_{1} s_{2}}$ & Binary parameters, if arrival-departure lines $s_{1}$ and $s_{2}$ are close to the same platform, $\delta_{s_{1}, s_{2}}=1$, else, $\delta_{s_{1}, s_{2}}=0$ \\
\hline$\vartheta_{c_{1} c_{2}}$ & Binary parameters, if there is transfer relationship between trains $c_{1}$ and $c_{2}, \vartheta_{c_{1}, c_{2}}=1$, else, $\vartheta_{c_{1}, c_{2}}=0$ \\
\hline$T_{m}^{1}$ & Headway time of trains which occupy the same arrival-departure lines \\
\hline$T_{m}^{2}$ & Minimum time interval of two trains at the potential conflict area \\
\hline$T_{m}^{3}$ & Minimum transfer time of passengers on the platform \\
\hline
\end{tabular}

\section{Mathematical Model of TRP in High-Speed Railway Station}

In this section, we put forward a mathematical model for TRP in a high-speed rail station. To depict this problem more clearly, the following discussion will concentrate on specifying each part of the models, including the objective functions and systematic constraints.

\subsection{Objective Function}

3.1.1. Utilization Balanced of the Arrival-Departure Lines. During railway operations, the challenge is to decrease the overall cost by means of a more efficient use of available resources as mentioned in Giacco et al. [23]. It is worth noting that the route is shortest if train is allotted on the sidings close to main lines. Otherwise, the distance would increase if the train is arranged to stop on others, especially the furthest arrival-departure line from the main line. If the objective is only to minimize the trains' total travel time, a large number of trains would like to stop at the arrivaldeparture lines near the main line, while the arrival-departure lines far from the main line are not occupied. If things go on like this it would cause serious wear and tear on the tracks if one arrival-departure line is often occupied repeatedly, which may bring extra costs owing to the frequent maintenance 
TABLE 2: Decision variables.

\begin{tabular}{ll}
\hline Notations & Description \\
\hline$x_{c s}$ & Binary variables, if the arrival-departure line $s$ is occupied by the train $c, x_{c s}=1$. Otherwise, $x_{c s}=0$ \\
$x_{c l}^{i}$ & Binary variables, if the route $l$ in the left bottleneck is occupied by the outbound train $c$ when pulls in, $x_{c l}^{i}=1$. \\
& Otherwise, $x_{c l}^{i}=1$ \\
$x_{c l}^{o}$ & Binary variables, if the route $l$ in the left bottleneck is occupied by the inbound train $c$ when departs from the \\
& station, $x_{c l}^{i}=1$. Otherwise, $x_{c l}^{i}=1$ \\
$x_{c r}^{i}$ & Binary variables, if the route $r$ in the right bottleneck is occupied by the inbound train $c$ when pulls in, $x_{c r}^{i}=1$. \\
$x_{c r}^{o}$ & Otherwise, $x_{c r}^{i}=1$ \\
$t_{c}$ & Binary variables, if the route $r$ in the right bottleneck is occupied by the outbound train $c$ when departs from the \\
$\delta_{c_{1}, c_{2}}$ & station, $x_{c r}^{o}=1$. Otherwise, $x_{c r}^{o}=1$ \\
& Integer variables, indicates the starting time of train $c$ to pull into the station \\
& Binary variables, indicates the trains $c_{1}$ and $c_{2}$ precedence relation at the same track. If the train $c_{1}$ precedes $c_{2}$, \\
& $\delta_{c_{1}, c_{2}}=1$. Otherwise, $\delta_{c_{1}, c_{2}}=0$
\end{tabular}

and repaired, the utilization proportionality of the arrivaldeparture lines should be kept when arranging trains' routes. The served trains provided by the arrival-departure lines include passing trains and stopping trains. As can be seen, the equilibrium is not only reflected in the number of trains, but also in the train's travel time that involves dwell time on the arrival-departure lines. It is converted into the mathematical expression and represented by the sum of variances of the number of trains and their dwell times:

$$
\begin{aligned}
\min z_{1} & \\
= & \frac{1}{m} \sum_{s \in S}\left(\sum_{c \in C} x_{c s}-\frac{n}{m}\right)^{2} \\
& +\frac{1}{m} \sum_{s \in S}\left(\sum_{c \in C} t_{c s} x_{c s}-\frac{\sum_{c \in C} t_{c s}}{m}\right)^{2} .
\end{aligned}
$$

In the objective function (13), $m$ is the number of arrivaldeparture lines and $n$ is the number of trains (i.e., the number of arrival-departure lines that are occupied). $\sum_{c \in C} t_{c s} x_{c s}$ denotes the sum of all trains' travel time on the arrivaldeparture lines. The first half of the function (13) shows the number of trains' occupancy balance and the second half means that the trains' travel time are relatively balanced on the arrival-departure lines.

3.1.2. Minimize the Trains' Total Travel Time. Trains' travel time on the tracks is another important factor in the optimization of the train routing problem. So the second optimization objective function is minimizing the trains' total travel time in the station:

$$
\begin{aligned}
\min & z_{2} \\
= & \sum_{s \in S} \sum_{l \in L} \sum_{r \in R} \sum_{c \in C_{1}} \alpha_{c}\left(x_{c l}^{i} t_{c s}^{l}+x_{c s} t_{c s}+x_{c r}^{o} t_{c s}^{l e}\right) \\
& +\sum_{s \in S} \sum_{l \in L} \sum_{r \in R} \sum_{c \in C_{2}} \alpha_{c}\left(x_{c r}^{i} t_{c s}^{r}+x_{c s} t_{c s}+x_{c l}^{o} t_{c s}^{l e}\right) .
\end{aligned}
$$

In (14), the front part indicates the total travel time of outbound trains $c \in C_{1}$; the last half expresses inbound trains $c \in C_{2}$ total travel time in the railway station. In order to ensure the satisfaction of passengers, the train reception and departure operations are punctuality and cannot be adjusted drastically. Compared to this, the shunting operations can be adjusted to be relatively flexible. At the same time, the punctuality requirements are different for different types of trains (passenger trains and freight trains). Therefore, the weight coefficient $\alpha_{c}$ is introduced to indicate the significance of each train.

\subsubsection{Minimize the Trains' Total Departure Time Deviations.} For railway operators, the punctuality of trains is necessary for the order and efficient operation of the station. Meanwhile, it is also the most concern of passengers. Therefore, the third optimization objective considered in this paper is minimizing the trains' total deviations between the trains' starting time to enter the station and the planned timetables of trains pulling in

$$
\min z_{3}=\sum_{c \in C} \alpha_{c}\left(t_{c}-t_{c}^{*}\right)
$$

In (15), the same $\alpha_{c}$ weight coefficient is quoted. $t_{c}$ is the starting time of train $c$ pulling into the station, and $t_{c}^{*}$ represents the planned entering time of trains which can calculate through pregiven timetable.

In summary, the linearly weighted compromise approach is adopted to handle the objective functions, and the objective function studied in this paper is

$$
\min Z=\beta_{1} z_{1}+\beta_{2} z_{2}+\beta_{3} z_{3}
$$

In the objective function (16), $\beta_{i}(i=1,2,3)$ are the weight coefficients with $0 \leq \beta_{i} \leq 1(i=1,2,3)$ and meet the equation requirement: $\beta_{1}+\beta_{2}+\beta_{3}=1$. It is set to distinguish the importance of different objectives. The specific values are given in the cases study. 

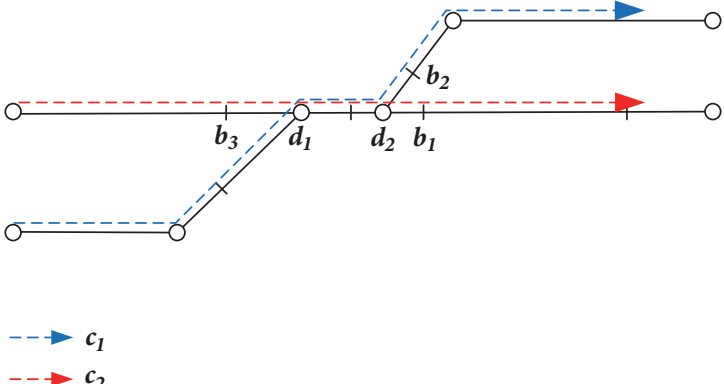
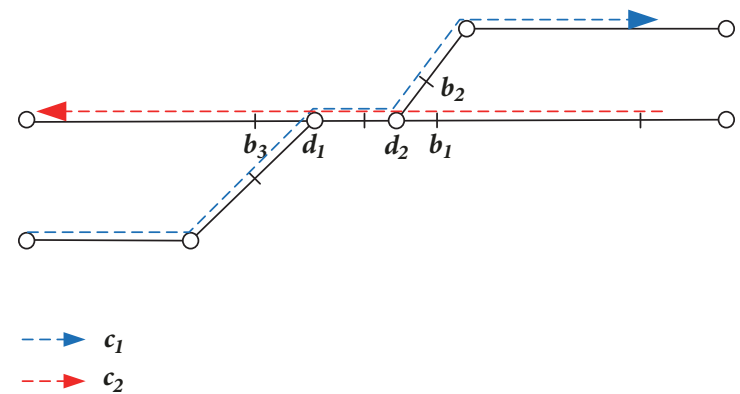

(a)

(b)

FIGURE 9: Conflict resolution when (a) two same direction trains' routing conflict, where (b) is a conflict of two reverse direction trains' routes.

\subsection{Constraints}

3.2.1. Constraints of Conflicts and Dispersion. There are two types of routing conflicts: on the overlap track segments of trains' routes and the potential conflicts at turnout nodes beside arrival-departure line. More detailed formulation of each set of constraints is provided as follows.

(1) Conflicts and Dispersion of Trains' Routes on Track Segments. The conflicts on the overlap track segments of trains' routes are discussed into the conflicts at left bottleneck, arrival-departure lines, and right bottleneck when trains go through the railway station. Here we build mathematical formulas to illustrate how to mitigate the conflicts.

If there is a conflict owing to the delay or interruption, that is, there would be hostile routes of trains, railway operators should take certain measures to ensure trains pass the hostile track segments orderly and efficiently. Figure 9 is a schematic diagram of routing conflicts of the same/reverse direction. The conflict occurs at track segment $d_{1} \sim d_{2}$ of trains $c_{1}$ and $c_{2}$, and track insulation joints $b_{1}, b_{2}$ and $b_{3}$. When the same direction conflicts take place (i.e., as shown in Figure 9(a)), if $c_{1}$ is arranged first, train $c_{2}$ can enter after the tail of train $c_{1}$ passing the insulation joint $b_{2}$ to suit the safety requirement. Similar, when the reverse direction conflict occurs as shown in Figure 9(b), train $c_{2}$ can enter after the tail of train $c_{1}$ passes the insulation joint $b_{2}$ if train $c_{1}$ is arranged first; train $c_{1}$ can enter after the tail of train $c_{2}$ passes the insulation joint $b_{2}$ if train $c_{2}$ is arranged first.

In accordance with the progress of the trains, each possible conflict is considered and stated as follows.

(i) Routes Conflicts at the Left Bottleneck. As described previously, we first discuss two outbound trains' routes which have overlapped tracks when arriving at the station from the left bottleneck. It should be formulated as $t_{c_{2}}-t_{c_{1}}-t_{c_{1} c_{2}}^{\text {con }} \geq 0$, or $t_{c_{1}}-t_{c_{2}}-t_{c_{2} c_{1}}^{c o n} \geq 0$, where $t_{c_{1} c_{2}}^{c o n}$ is the time from train $c_{1}$ starting pulling into the station to the tail of it passing through the insulation joint $b_{2}$, i.e., the time interval of trains to enter the railway station. The value can be obtained by (1). Similarly, $t_{c_{2} c_{1}}^{c o n}$ is the time from train $c_{2}$ starting entering to the tail of it passing through the insulation joint $b_{1}$.
Considering the order and relationship between trains and routes which are chosen, the constraints can be expressed as

$$
\begin{aligned}
& x_{c_{1} l_{1}}^{i} x_{c_{2} l_{2}}^{i} \theta_{l_{1} l_{2}}\left(t_{c_{2}}-t_{c_{1}}-t_{c_{1} c_{2}}^{c o n}+\right.\left.M\left(1-\delta_{c_{1}, c_{2}}\right)\right) \geq 0, \\
& \forall c_{1}, c_{2} \in C_{1}, l_{1}, l_{2} \in L, \\
& x_{c_{1} l_{1}}^{i} x_{c_{2} l_{2}}^{i} \theta_{l_{1} l_{2}}\left(t_{c_{1}}-t_{c_{2}}-t_{c_{2} c_{1}}^{c o n}+\mathrm{M} \delta_{c_{1}, c_{2}}\right) \geq 0, \\
& \forall c_{1}, c_{2} \in C_{1}, l_{1}, l_{2} \in L .
\end{aligned}
$$

In the constraints (17) and (18), $\delta_{c_{1}, c_{2}}$ is a binary variable that indicates the trains' precedence relationship passing through the overlapping tracks. If train $c_{1}$ precedes train $c_{2}, \delta_{c_{1}, c_{2}}=1$. Otherwise, $\delta_{c_{1}, c_{2}}=0 . M$ is a sufficiently large number. Not only the safety time interval but also the routes' selection relationship are taken into account. So in constraints (17) and (18), if a route $l_{1}$ is chosen in the left bottleneck by outbound train $c_{1}\left(x_{c_{1} l_{1}}^{i}=1\right.$, else, $\left.x_{c_{1} l_{1}}^{i}=0\right)$ and a route $l_{2}$ is occupied by outbound train $c_{2}$ to enter the station $\left(x_{c_{2} l_{2}}^{i}=1\right.$, else, $\left.x_{c_{2} l_{2}}^{i}=0\right)$ and, in the meantime, there are spatial conflicts between routes $l_{1}$ and $l_{2}$ (if there are spatial conflicts between routes, $\theta_{l_{1} l_{2}}=1$. Otherwise, $\theta_{l_{1} l_{2}}=0$.), then the safe time interval $t_{c_{1} c_{2}}^{c o n}$ should be held between entrance time of trains $c_{1}$ and $c_{2}$.

Secondly, the situation for two inbound trains that depart from the station through the left bottleneck is similar. That is, after the front train leaving the station, the latter train can depart from the arrival-departure lines through the left bottleneck after the safe time interval $t_{c_{1} c_{2}}^{\text {con }} \cdot t_{c_{2}}^{\text {fend }}-t_{c_{1}}^{\text {fend }}-t_{c_{1} c_{2}}^{\text {con }} \geq$ 0 or $t_{c_{1}}^{\text {fend }}-t_{c_{2}}^{\text {fend }}-t_{c_{2} c_{1}}^{\text {con }} \geq 0$ must hold which are equivalent to the following constraints:

$$
\begin{aligned}
& x_{c_{1} l_{1}}^{i} x_{c_{2} l_{2}}^{i} \theta_{l_{1} l_{2}}\left(t_{c_{2}}^{\text {fend }}-t_{c_{1}}^{\text {fend }}-t_{c_{1} c_{2}}^{\text {con }}+M\left(1-\delta_{c_{1}, c_{2}}\right)\right) \\
& \geq 0, \quad \forall c_{1}, c_{2} \in C_{2}, l_{1}, l_{2} \in L, \\
& x_{c_{1} l_{1}}^{i} x_{c_{2} l_{2}}^{i} \theta_{l_{1} l_{2}}\left(t_{c_{1}}^{\text {fend }}-t_{c_{2}}^{\text {fend }}-t_{c_{2} c_{1}}^{\text {con }}+\mathrm{M} \delta_{c_{1}, c_{2}}\right) \geq 0, \\
& \forall c_{1}, c_{2} \in C_{2}, l_{1}, l_{2} \in L .
\end{aligned}
$$


The occupation ending time on the arrival-departure lines can be calculated by (6) and (12), which is $t_{c}^{\text {fend }}=t_{c}+$ $t_{c}^{\text {setup }}+t_{c s}^{r}+t_{c s}$.

Thirdly, for two opposite direction trains, an outbound train $c_{1}$ would arrive at the station through the left bottleneck while an inbound train $c_{2}$ would depart from the arrivaldeparture lines; then they may cause route conflicts which can be avoided by the following constraints:

$$
\begin{array}{r}
x_{c_{1} l_{1}}^{i} x_{c_{2} l_{2}}^{i} \theta_{l_{1} l_{2}}\left(t_{c_{2}}^{\text {fend }}-t_{c_{1}}-t_{c_{1} c_{2}}^{c o n}+M\left(1-\delta_{c_{1}, c_{2}}\right)\right) \geq 0, \\
\forall c_{1} \in C_{1}, c_{2} \in C_{2}, l_{1}, l_{2} \in L, \\
x_{c_{1} l_{1}}^{i} x_{c_{2} l_{2}}^{i} \theta_{l_{1} l_{2}}\left(t_{c_{1}}-t_{c_{2}}^{\text {fend }}-t_{c_{2} c_{1}}^{c o n}+M \delta_{c_{1}, c_{2}}\right) \geq 0, \\
\forall c_{1} \in C_{1}, c_{2} \in C_{2}, l_{1}, l_{2} \in L .
\end{array}
$$

(ii) Routes Conflicts at the Right Bottleneck. Similar to the conflicts in the left bottleneck, the trains routing conflicts for two inbound trains, two outbound trains, and two opposite direction trains should satisfy the following constraints in the right bottleneck.

When two inbound trains arrive at the station through the right bottleneck, the route conflicts may occur. Therefore, we set following constraints to avoid collision:

$$
\begin{array}{r}
x_{c_{1} r_{1}}^{i} x_{c_{2} r_{2}}^{i} \theta_{r_{1} r_{2}}\left(t_{c_{2}}-t_{c_{1}}-t_{c_{1} c_{2}}^{c o n}+\right. \\
\left.\forall\left(1-\delta_{c_{1}, c_{2}}\right)\right) \geq 0, \\
\forall c_{1}, c_{2} \in C_{2}, r_{1}, r_{2} \in R, \\
x_{c_{1} r_{1}}^{i} x_{c_{2} r_{2}}^{i} \theta_{r_{1} r_{2}}\left(t_{c_{1}}-t_{c_{2}}-t_{c_{2} c_{1}}^{c o n}+M \delta_{c_{1}, c_{2}}\right) \geq 0, \\
\forall c_{1}, c_{2} \in C_{2}, r_{1}, r_{2} \in R .
\end{array}
$$

For two outbound trains which would depart from the station through the right bottleneck

$$
\begin{array}{r}
x_{c_{1} r_{1}}^{i} x_{c_{2} r_{2}}^{i} \theta_{r_{1} r_{2}}\left(t_{c_{2}}^{f}-t_{c_{1}}^{f}-t_{c_{1} c_{2}}^{c o n}+\mathrm{M}\left(1-\delta_{c_{1}, c_{2}}\right)\right) \geq 0, \\
\forall c_{1}, c_{2} \in C_{1}, r_{1}, r_{2} \in R, \\
x_{c_{1} r_{1}}^{i} x_{c_{2} r_{2}}^{i} \theta_{r_{1} r_{2}}\left(t_{c_{1}}^{f}-t_{c_{2}}^{f}-t_{c_{2} c_{1}}^{c o n}+M \delta_{c_{1}, c_{2}}\right) \geq 0, \\
\forall c_{1}, c_{2} \in C_{1}, r_{1}, r_{2} \in R .
\end{array}
$$

For two opposite direction trains, considering the order and relationship between trains and routes which are chosen, the constraint can be

$$
\begin{array}{r}
x_{c_{1} r_{1}}^{i} x_{c_{2} r_{2}}^{i} \theta_{r_{1} r_{2}}\left(t_{c_{2}}-t_{c_{1}}^{\text {fend }}-t_{c_{1} c_{2}}^{\text {con }}+\mathrm{M}\left(1-\delta_{c_{1}, c_{2}}\right)\right) \geq 0, \\
\forall c_{1} \in C_{1}, c_{2} \in C_{2}, r_{1}, r_{2} \in R, \\
x_{c_{1} r_{1}}^{i} x_{c_{2} r_{2}}^{i} \theta_{r_{1} r_{2}}\left(t_{c_{1}}^{\text {fend }}-t_{c_{2}}-t_{c_{2} c_{1}}^{c o n}+M \delta_{c_{1}, c_{2}}\right) \geq 0, \\
\forall c_{1} \in C_{1}, c_{2} \in C_{2}, r_{1}, r_{2} \in R .
\end{array}
$$

Among these six constraints, the safe time interval $t_{c_{1} c_{2}}^{c o n}$ should be kept. The same with the discussion above, the occupation end time on the arrival-departure lines can be calculated by (6) and (12).

(iii) Routes Conflicts on the Arrival-Departure Lines. The train routing conflicts which may occur on the arrival-departure lines has been described in Section 2. Here we propose the algebraic formulas to resolve the conflicts. If the difference of two trains' departure time is smaller than the headway time $T_{m}^{1}$, in other words, if train $c_{1}$ occupies the arrival-departure line first, train $c_{2}$ can enter from bottleneck after the end of occupied time at arrival-departure lines of train $c_{1}$ and after a headway time: $\left(t_{c_{2}}^{f}-t_{c s}^{s}-t_{c s}^{d}\right)-\left(t_{c_{1}}^{f}+t_{c s}^{a}\right)-T_{m}^{1} \geq 0$, where $t_{c}^{f}=t_{c l}^{\text {end }}+t_{c s}^{d}+t_{c s}^{s}$ for the outbound trains and for the inbound trains $t_{c}^{f}=t_{c r}^{e n d}+t_{c s}^{d}+t_{c s}^{s}$ which can be calculated according to (9) and (10) described in Section 2. According to Assumption 1, the additional acceleration and deceleration time of trains that run in different speed are same, and the formula is converted to $t_{c_{2}}^{f}-t_{c_{1}}^{f}-t_{c_{2} s}-T_{m}^{1} \geq 0$. Similar, if train $c_{2}$ enters first, it should be satisfied: $t_{c_{1}}^{f}-t_{c_{2}}^{f}-t_{c_{1} s}-T_{m}^{1} \geq$ 0 .

Thus the constraints should be satisfied as follows:

$$
\begin{array}{r}
x_{c_{1} s} x_{c_{2} s}\left(t_{c_{2}}^{f}-t_{c_{1}}^{f}-t_{c_{2} s}-T_{m}^{1}+\mathrm{M}\left(1-\delta_{c_{1}, c_{2}}\right)\right) \geq 0, \\
\forall c_{1}, c_{2} \in C, l_{1}, l_{2} \in L, s \in S \\
x_{c_{1} s} x_{c_{2} s}\left(t_{c_{1}}^{f}-t_{c_{2}}^{f}-t_{c_{1} s}-T_{m}^{1}+M \delta_{c_{1}, c_{2}}\right) \geq 0, \\
\forall c_{1}, c_{2} \in C, l_{1}, l_{2} \in L, s \in S .
\end{array}
$$
(10).

The value of $t_{c}^{f}$ can be calculated from (3), (6), (9), and

(2) Potential Conflicts and Avoidance. In small scale stations, there are a small number of arrival-departure lines and platforms that any of them can be occupied by trains. As shown in Figure 5, the outbound train $c_{1}$ may occupy the arrival-departure line 3 to stop when the operation is busy. In this case, it may cause potential conflicts between opposite direction trains if they choose the arrival-departure lines on the same side. Thus measures should be adopted to avoid the two trains arriving the potential conflicting area simultaneously. The trains' order should be determined by the decision variable $\delta_{c_{1}, c_{2}}$. In Figure 5(a), if train $c_{1}$ arrives at the station through the left bottleneck, train $c_{2}$ can depart from the arrival-departure line 3 after the tail of train $c_{1}$ passes the potential conflicting area after a safety time interval $T_{m}^{2}$ (i.e., the inequality $t_{c_{2}}^{f}-t_{c_{1} l}^{\text {end }}-T_{m}^{2} \geq 0$ should be satisfied). Similar, if train $c_{2}$ leaves the station first, after a safety time interval, train $c_{1}$ can enter the station through the left bottleneck after the tail of train $c_{2}$ passes the potential conflicting area (i.e., satisfying $t_{c_{1}}-t_{c_{2}}^{\text {fend }}-T_{m}^{2} \geq 0$ ). 
To sum up, we use the following formula to avoid potential conflicts:

$$
\begin{array}{r}
x_{c_{1} l}^{i} x_{c_{2} s} \gamma_{c_{1} c_{2}}\left(t_{c_{2}}^{f}-t_{c_{1} l}^{e n d}-T_{m}^{2}+M\left(1-\delta_{c_{1}, c_{2}}\right) \geq 0,\right. \\
\forall c_{1} \in C_{1}, c_{2} \in C_{2}, l \in L, s \in S, \\
x_{c_{1} l}^{i} x_{c_{2} s} \gamma_{c_{1} c_{2}}\left(t_{c_{1}}-t_{c_{2}}^{f e n d}-T_{m}^{2}+M \delta_{c_{1}, c_{2}}\right) \geq 0, \\
\forall c_{1} \in C_{1}, \quad c_{2} \in C_{2}, l \in L, s \in S .
\end{array}
$$

It is suitable for solving the potential conflicts as shown in Figure 5(a). Constraints (33) and (34) are used to solve the problems of the situation in Figure 5(b):

$$
\begin{array}{r}
x_{c_{1} r}^{i} x_{c_{2} s} \gamma_{c_{1} c_{2}}\left(t_{c_{2}}-t_{c_{1}}^{\text {fend }}-T_{m}^{2}+M\left(1-\delta_{c_{1}, c_{2}}\right) \geq 0,\right. \\
\forall c_{1} \in C_{1}, c_{2} \in C_{2}, r \in R, s \in S, \\
x_{c_{1} r}^{i} x_{c_{2} s} \gamma_{c_{1} c_{2}}\left(t_{c_{1}}^{f}-t_{c_{2} r}^{e n d}-T_{m}^{2}+M \delta_{c_{1}, c_{2}}\right) \geq 0, \\
\forall c_{1} \in C_{1}, c_{2} \in C_{2}, r \in R, s \in S .
\end{array}
$$

3.2.2. The Limited Capacity Restrictions. This constraint set considers the limited infrastructure capacity. Obviously, a train can only utilize one route at bottleneck and arrivaldeparture lines. To guarantee the safe operation of trains traveling in the railway station, the constraints are listed as follows:

$$
\begin{array}{ll}
\sum_{s \in S} x_{c s}=1 & \forall c \in C, \\
\sum_{l \in L} x_{c l}^{i}=1 & \forall c \in C_{1}, \\
\sum_{r \in R} x_{c r}^{o}=1 \quad \forall c \in C_{1}, \\
\sum_{r \in R} x_{c r}^{i}=1 \quad \forall c \in C_{2}, \\
\sum_{l \in L} x_{c l}^{o}=1 \quad \forall c \in C_{2} .
\end{array}
$$

Constraint (35) ensures that only one arrival-departure line can be occupied by one train. Constraints (36) and (37) guarantee that each train must utilize one and only one route to traverse the left and right bottleneck for each outbound train, respectively. Similarly, only one route in the right and left bottleneck can be chosen by each inbound train through constraints (38) and (39), respectively.

3.2.3. Connectivity of Train Routing Constraints. Trains' routing connectivity should be ensured. That is, for an outbound train, the route occupied at the left bottleneck should connect to the arrival-departure line which must connect to the route in the right bottleneck. Otherwise, it is conceivable that the train cannot pass the discontinuous route through the station. Therefore, these restrictions can guarantee the feasibility of trains' routes.

$$
\begin{aligned}
& x_{c s}=\sum_{l \in R_{s}^{l}} x_{c l}^{i}, \quad \forall c \in C_{1}, s \in S, l \in L, \\
& x_{c s}=\sum_{l \in R_{s}^{l}} x_{c l}^{o}, \quad \forall c \in C_{2}, s \in S, l \in L, \\
& x_{c s}=\sum_{r \in R_{s}^{r}} x_{c r}^{i}, \quad \forall c \in C_{2}, \quad s \in S, \quad r \in R, \\
& x_{c s}=\sum_{r \in R_{s}^{r}} x_{c r}^{o}, \quad \forall c \in C_{1}, \quad s \in S, \quad r \in R,
\end{aligned}
$$

where $R_{s}^{l}$ and $R_{s}^{r}$ are sets of left and right bottleneck routes connected to the arrival-departure line $s$ respectively.

3.2.4. Restrictions of Passengers' Transfer at Station. It represents one of the concerns of railway operators to facilitate passengers transfer to improve the service level. Nowadays, various large scale high-speed rail stations have set up shortcut channels in the railway station to offer convenience for passengers' transfer in station. This enables passengers to achieve their traveling purpose in a shorter time and cost. There are transfer elevators at each platform in some large and well-equipped facilities stations. And passengers can quickly find the corresponding ticket gate directly through the information from the ticket or the electronic screen.

Two trains that may have transfer relationship are discussed in this paper. A constraint is set to keep the two trains stopped at two near platforms to ensure that passengers or crews transfer in a comfortable time:

$$
x_{c_{1} s_{1}} x_{c_{2} s_{2}} \delta_{s_{1} s_{2}} \vartheta_{c_{1} c_{2}} \geq 1, \quad \forall c_{1}, c_{2} \in C, s_{1}, s_{2} \in S
$$

At the same time, the target train should stop at the platform for a time interval $T_{m}^{3}$ to ensure that all passengers complete transfer comfortably, which is required to allow passengers alight from one train, move to the corresponding platform track, and board the other train. So we obtain the following constraints:

$$
\begin{array}{r}
\vartheta_{c_{1} c_{2}} x_{c_{1} s_{1}} x_{c_{2} s_{2}}\left(t_{c_{2}}^{f}-\left(t_{c_{1}}^{f}-t_{c_{1} s}^{s}\right)-T_{m}^{3}+M\left(1-\delta_{c_{1}, c_{2}}\right)\right) \\
\geq 0, \quad \forall c_{1}, c_{2} \in C, s_{1}, s_{2} \in S, \\
\vartheta_{c_{1} c_{2}} x_{c_{1} s_{1}} x_{c_{2} s_{2}}\left(t_{c_{1}}^{f}-\left(t_{c_{2}}^{f}-t_{c_{2} s}^{s}\right)-T_{m}^{3}+M \delta_{c_{1}, c_{2}}\right) \geq 0, \\
\forall c_{1}, c_{2} \in C, s_{1}, s_{2} \in S .
\end{array}
$$


3.2.5. Variable Feasible Ranges. The following constraints indicate the feasible ranges of the variables:

$$
\begin{gathered}
t_{c}-t_{c}^{*} \geq 0, \quad \forall c \in C, \\
t_{c} \in Z, \quad \forall c \in C, \\
x_{c s}, x_{c l}^{i}, x_{c l}^{o}, x_{c r}^{i}, x_{c r}^{o} \in\{0,1\}, \\
s \in S, c \in C, l \in L, r \in R, \\
\delta_{c_{1}, c_{2}} \in\{0,1\}, \quad c \in C .
\end{gathered}
$$

Constraint (47) indicates that all trains cannot depart from the station before the planned timetable, where integer decision variable $t_{c}$ indicates the start time measured in minutes to enter the station of train $c$. Constraints (49) and (50) ensure that those decision variables are $0-1$ variables.

Herein, the train routing problem for heterogeneous trains can be formulated as the following model, which is essentially a mixed integer nonlinear programming model:

$$
\begin{aligned}
& \min Z=\beta_{1} z_{1}+\beta_{2} z_{2}+\beta_{3} z_{3}, \\
& \text { s.t. } \quad \text { constraints }(17)-(50) .
\end{aligned}
$$

\section{Solution Methodology Based on Tabu Search}

The number of decision variables of the model will increase greatly as the increasing number of optional routes of trains which is NP-hard problem mentioned in Ahuja et al. [18] and Carey et al. [19]. In the proposed model, the situation scale of the values of variables $x$ is huge as the number of them is great. For instance, when $C=\left\{c_{1}, c_{2}\right\}$ and $L=\left\{l_{1}, l_{2}, l_{3}\right\}$, there are $2^{6}$ kinds of values of $x=\left[x_{c_{1} l_{1}}^{i}, x_{c_{1} l_{2}}^{i}, x_{c_{1} l_{3}}^{i}, x_{c_{2} l_{1}}^{i}, x_{c_{2} l_{2}}^{i}, x_{c_{2} l_{3}}^{i}\right]$, where each element of $x$ may take 0 or 1 . It would cost a significant amount of time to solve the problem under a larger case and may not even get the optimal solution in a reasonable computation time. Apart from this, the mathematic model is nonlinear and there is no algorithm which can solve such problems accurately at present. Therefore in this section, we design a tabu search algorithm stepwise to obtain the nearoptimal solution based on the nonlinear characteristic of the model for the TRP with a large scale railway station.

The tabu search algorithm is a deterministic metaheuristic on account of local search [24], which makes extensive use of memory for guiding the search. From the incumbent solution, non-tabu moves define a set of solutions which called the neighborhood of the incumbent solution. The best solution is selected as the new incumbent solution at each step and stored in the tabu list to avoid being trapped in local optima and re-visiting the same solutions. When the number of solutions achieves the length of the tabu list, the earliest one which entered the list is released. The algorithm stops until reaching the termination conditions.

In our model, we notice that constraints (17)-(34) and constraints (45) and (46) are nonlinear. It is obvious that if the variable $x$ is determined, the model (51) would become an integer linear programming model that determines the departure time of each train. For such mathematical programming model, there are already mature algorithms, such as branch and bound and cutting plane, etc. Taking constraints (17) and (18) as an example, the decision variables $x_{c_{1} l_{1}}^{i}$ and $x_{c_{2} l_{2}}^{i}$ are binary variables, and $\theta_{l_{1} l_{2}}$ is known binary parameters according to wether there are conflicts between routes $l_{1}$ and $l_{2}$. There are two cases due to the value of decision variables. The first is that the value of any variables or parameter is 0 among the decision variables $x_{c_{1} l_{1}}^{i}, x_{c_{2} l_{2}}^{i}$ and parameter $\theta_{l_{1} l_{2}}$. Then the left side of the formula is 0 , and the constraint is effective apparently. The second case is that the values of them are 1 , then the constraints were satisfied if the formulas are satisfied inside the brackets. Hence the inequality constraints are obviously satisfied in the first case. And in the second case, the constraints turn into an integer linear constraints, not only for constraints (17) and (18), but for other constraints in the model (51). As a result, we set the decision variable $x$ as the tabu object. Based on that, through generating neighborhood of incumbent solution and searching a good solution at each step, we can gradually approach the near-optimal solution.

To deal with the problems mentioned above in the model presented in this paper, we discuss the setting of the neighborhood of incumbent solution, the selection of initial solution, and the scheme of algorithm in detail below.

4.1. Neighborhood of Incumbent Solution. The establishment of incumbent solution neighborhood is crucial for a better direction of the search, which affects the quality of solution at each step. In our model, we hope that trains would have the shortest travel time, which performances the shortest routes. As mentioned, the travel distance would increase if trains are arranged on the sidings far from the main line. In our problem, we need to determine a good quality initial solution and tabu move to reach the near-optimal solution. Therefore we generate two different neighborhoods of the incumbent solution, respectively:

$N_{\text {initial }}$ : based on the incumbent routing set, which contains routes in bottleneck and arrival-departure line, the two adjacent sidings and corresponding routes in bottleneck are accommodated into it. That is, the neighborhood contains not only the incumbent arrival-departure lines of trains and corresponding routes in left and right bottleneck, but also the two adjacent sidings and corresponding routes in the bottleneck.

$N_{\text {tabu }}$ : the neighborhood of incumbent routing set for tabu move contains $N_{\text {initial }}$, which involves four adjacent siding and corresponding routes in bottleneck. Besides, remove the situation that trains with close departure times and occupy the same sidings.

In general, $N_{\text {initial }}$ is used to determine a good quality initial solution, whose scale is smaller. And we need to search the solution roughly in a short time. In contrast to this, $N_{t a b u}$ is a wide neighborhood in order to avoid empty 


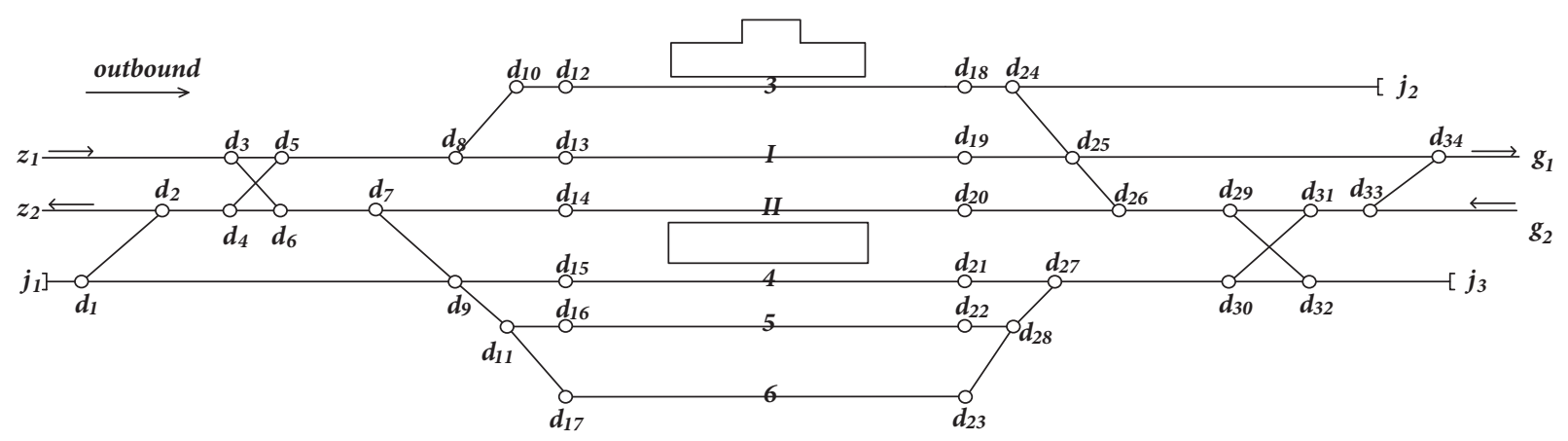

FIGURE 10: The structure of a small-scale station.

neighborhoods as far as possible while avoiding to search in all possible situations. It is thus worthwhile distinguishing the different neighborhood structures.

4.2. Determined of Initial Solution. As describe above, the quality of initial solution is crucial for the performance of tabu search. A good initial feasible solution can greatly improve the speed of searching for the optimal solution. If we randomly assign a set of routes as the incumbent solution, it would take a lot of time to search, and the quality of the near-optimal solution cannot be guaranteed. The objective value is much smaller obviously than the previous strategy if starting from a good incumbent solution. So in this paper, we can get a feasible solution of train routing set through our prior knowledge of trains' character and construction of the railway station which is just a preliminary program. In view of this, the corresponding routing set solution is selected which satisfies constraints and at the same time reaches the minimum objective function in the solution neighborhoods at next step. Then regard this solution as a new incumbent solution. By cycling this step until the iterative termination condition is satisfied, a batter initial solution of the tabu search algorithm is determined. The procedure is summarized in Table 3.

4.3. The Algorithm Scheme. In this paper, we first obtain the initial routes based on method describe in Table 3. Then we generate the neighborhood $N_{t a b u}$ of the incumbent solution to reduce the range of the search. And we solve the corresponding MLP in the $N_{t a b u}$ to choose the best solution. Finally, the best solution is chosen as the new incumbent solution and stored in the tabu list. The steps shown in Table 3 are repeated in sequence recursively. We summarize the procedure of this heuristic algorithm in Table 4.

In this case the tabu list contains the situation of all trains' routes. The aspiration criterion is set to reinforce the local search to avoid losing an admirable state. That is, if a solution in the tabu list is largely superior to others, then reconsider and search it as a new incumbent solution. The other solutions are forbidden in the tabu list to avoid being trapped in local optima or infinite loops.

\section{Numerical Experiments}

In this section, two sets of numerical experiments are implemented to show the performance of our proposed model. Specifically, for the models formulated, a small case is implemented to demonstrate the application and performance, in which CPLEX solver is used to obtain exact optimal solution which compares with the near-optimal solution obtained by the proposed heuristic algorithm based on the tabu search, while in large scale case experiment, we apply the proposed heuristic algorithm to the Jinan West high-speed railway station, in which a tabu search algorithm is designed in PYTHON 2.7.13 to obtain the near-optimal solutions of trains routing on a Windows 10 platform with Intel(R) Core(TM) i7-8550U CPU and 8G RAM.

5.1. A Small-Scale Case Study. In this case, we consider a two main $I$ and $I I$ lines railway station as shown in Figure 10 which is outbound and inbound direction, respectively. In the left bottleneck, there are a reception line $z_{1}$ of outbound trains, a departure line $z_{2}$ of inbound trains, and a locomotive waiting track $j_{1}$. At the same time, there are 6 arrival-departure lines, of which arrival-departure lines II, 3 and 4 next to a platform. In the right bottleneck, there are 4 lines including a departure line $g_{1}$ of outbound trains, a reception line $g_{2}$ of inbound trains. and two locomotive waiting tracks $j_{2}$ and $j_{3}$. The distances between connection points in this railway station are shown in Table 5.

In order to test the effectiveness of the proposed models and solution algorithms, we derive a set of instances in this small-scale rail station with different numbers of trains (the type of trains is exhaustive to demonstrate the versatility and correctness of the model). Table 6 shows the origin and destination of each train, as well as the characteristic parameters of them. Trains $c_{1}$ and $c_{4}$ are outbound trains, while $c_{3}$ is inbound train. At the same time, there is a transfer relationship between $c_{1}$ and $c_{3}$. Trains $c_{2}$ and $c_{5}$ are shunting operations, wherein $c_{2}$ travels from the locomotive waiting tracks $j_{2}$ to $j_{1}$ and $c_{5}$ travels from $j_{1}$ to be an originating train (i.e., train $c_{5}$ would choose an arrival-departure line to stop and then depart from the station through the departure node $\left.g_{1}\right)$. In order to distinguish the importance of operations, the higher punctuality required for train reception, and 
TABLE 3: The scheme of determining initial solution.

Step 1. Input initial information, including trains' character, railway station parameters, train speed profiles, trains with transfer relationship, planned timetable, etc., set $\boldsymbol{i}=\mathbf{0}$.

Step 2. Choose a feasible routes set as incumbent solution $\boldsymbol{x}_{\boldsymbol{i n c}}=\boldsymbol{x}_{\boldsymbol{f}}$ through prior knowledge of trains and parameters of the railway station, get objective value $\boldsymbol{s} \boldsymbol{x}_{\boldsymbol{i n c}}$.

Step 3. Generate neighborhood $N_{\text {initial }}\left(x_{i n c}\right)$ of $x_{i n c}$, and corresponding neighborhood solution: $\operatorname{SN} N_{\text {initial }}\left(\boldsymbol{x}_{\text {inc }}\right)$.

Step 4. Search the best solution $s x^{*}$ in $S N_{\text {initial }}\left(x_{i n c}\right)$ and corresponding routing set $x^{*}$

Step 5. If the objective value of $s x^{*}$ is better than that of $s x_{i n c}, s x_{i n c}=s x^{*}, x_{i n c}=x^{*}$; otherwise, let $x_{i n c}$ as current solution continuously.

Step 6. If the value of $\boldsymbol{x}_{\text {inc }}$ does not change, $\boldsymbol{i}+=\mathbf{1}$; otherwise, go to step 7.

Step 7. If $\boldsymbol{i}==\mathbf{1}$, output $\boldsymbol{s} \boldsymbol{x}_{\boldsymbol{i n c}}$ and the corresponding routing set $\boldsymbol{x}_{\boldsymbol{i n c}}$, stop. Else, go to step 3.

TABLE 4: Pseudocode of the tabu search algorithm.

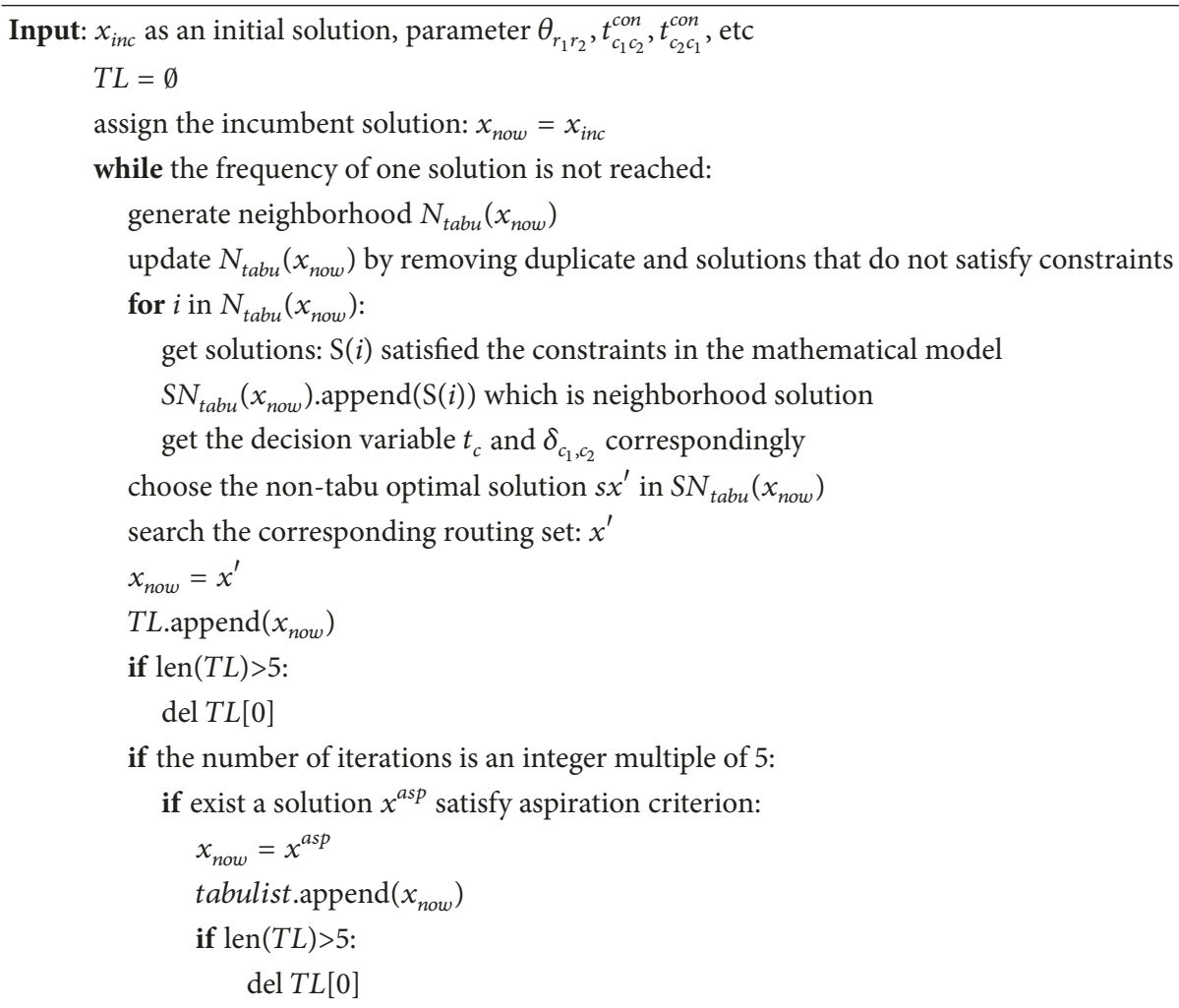

Output: optimal solution: $x_{\text {now }}, t_{c}$ and $\delta_{c_{1}, c_{2}}$

departure operations compared to the shunting operations, we set the weights to 0.9 and 0.1 , respectively. As for the multiobjective of our model, we attach the most importance to the punctuality, then the total travel time and utilization balanced of the arrival-departure lines are considered evenly. Thus set the parameters $\beta_{1}=\beta_{2}=0.3$ and $\beta_{3}=0.4$. In addition to this, the preparation time of each train is $1 \mathrm{~min}$, and the additional time that provides trains' acceleration and deceleration is $2 \mathrm{~min}$ and $1 \mathrm{~min}$, respectively.

We assume the headway time $T_{m}^{1}=2 \mathrm{~min}$, the minimum time interval of potential area of two trains $T_{m}^{2}=1 \mathrm{~min}$, and the minimum transfer time offered to passengers in the arrival-departure lines $T_{m}^{3}=10 \mathrm{~min}$. It is easy to neglect in this small example that if two opposite direction trains pass through node $d_{9}$ or $d_{27}$ one after the other, there may be potential conflicts.

The algorithm designed in Section 4 is used to solve the model of this small case. First, an incumbent solution of trains' routing is chosen based on the scheme described in Table 3, whose quality has a great influence on the efficiency of searching the near-optimal solution. The prior knowledge we considered here includes our analysis of the station structure, the nature of each train, and the route by which the train is most likely to occupy, etc. For instance, there is a transfer relationship between $c_{1}$ and $c_{3}$, while the arrival-departure lines $I I$ and 4 are located on either side of the platform. Therefore, we can arrange arrival-departure lines II and 4, respectively, as initial routes of trains $c_{1}$ and $c_{3}$. In terms of 
TABLE 5: Distances between points in the railway network in Figure 10.

\begin{tabular}{|c|c|c|c|c|c|c|c|c|}
\hline Node: $d_{i}$ & Node: $d_{i}$ & Distance $(\mathrm{m})$ & Node: $d_{i}$ & Node: $d_{i}$ & Distance $(\mathrm{m})$ & Node: $d_{i}$ & Node: $d_{i}$ & Distance $(\mathrm{m})$ \\
\hline 1 & 2 & 90 & 9 & 11 & 60 & 22 & 28 & 85 \\
\hline 1 & 9 & 305 & 10 & 12 & 80 & 23 & 28 & 90 \\
\hline 2 & 4 & 60 & 11 & 16 & 85 & 24 & 25 & 90 \\
\hline 3 & 5 & 48 & 11 & 17 & 90 & 25 & 34 & 300 \\
\hline 3 & 6 & 60 & 12 & 18 & 750 & 25 & 26 & 60 \\
\hline 4 & 5 & 60 & 13 & 19 & 750 & 26 & 29 & 100 \\
\hline 4 & 6 & 48 & 14 & 20 & 750 & 27 & 30 & 148 \\
\hline 5 & 8 & 175 & 15 & 21 & 750 & 28 & 27 & 60 \\
\hline 6 & 7 & 100 & 16 & 22 & 750 & 29 & 31 & 60 \\
\hline 7 & 14 & 170 & 17 & 23 & 750 & 29 & 32 & 90 \\
\hline 7 & 9 & 90 & 18 & 24 & 80 & 30 & 31 & 90 \\
\hline 8 & 10 & 90 & 19 & 25 & 125 & 30 & 32 & 60 \\
\hline 8 & 13 & 125 & 20 & 26 & 170 & 31 & 33 & 50 \\
\hline 9 & 15 & 125 & 21 & 27 & 125 & 33 & 34 & 60 \\
\hline
\end{tabular}

TABLE 6: The parameters of trains in the small case.

\begin{tabular}{lcccccc}
\hline Train & Origin & Destination & $\begin{array}{c}\text { Average velocity } \\
(\mathrm{km} / \mathrm{h})\end{array}$ & Operations & $\begin{array}{c}\text { Planned } \\
\text { timetable }\end{array}$ & $\begin{array}{c}\text { Stop time } \\
(\mathrm{min})\end{array}$ \\
\hline$c_{1}$ & $z_{1}$ & $g_{1}$ & 30 & Passing & $11: 25$ & 17 \\
$c_{2}$ & $j_{2}$ & $j_{1}$ & 15 & Shunting & $11: 28$ & 0 \\
$c_{3}$ & $g_{2}$ & $z_{2}$ & 30 & Passing & $11: 30$ & 10 \\
$c_{4}$ & $z_{1}$ & $g_{1}$ & 30 & Passing & $11: 34$ & 6 \\
$c_{5}$ & $j_{1}$ & $g_{1}$ & 15 & Shunting & $11: 37$ & 30 \\
\hline
\end{tabular}

TABLE 7: The start time of trains to enter the station.

\begin{tabular}{lccccc}
\hline Train & $c_{1}$ & $c_{2}$ & $c_{3}$ & $c_{4}$ & $c_{5}$ \\
Start time & $11: 25$ & $11: 28$ & $11: 30$ & $11: 34$ & $11: 37$ \\
Delay time $(\mathrm{min})$ & 0 & 0 & 0 & 0 & 0 \\
\hline
\end{tabular}

the origin and destination of train $c_{2}$, there is only one route. And for the remaining two trains, they can be organized to stop at the arrival-departure lines close to the entrance line, as $c_{4}$ occupies arrival-departure line $I$ and $c_{5}$ would stop at arrival-departure line 4.

At the same time, there are 5 trains stopping and passing through the railway station. In the case that origin and destination are prespecified, the number of each train's route at left bottleneck, arrival-departure line, and right bottleneck is 6 , respectively. Therefore, there are $6^{5}$ possibilities for the routing arrangement of 5 trains. Of course, it may increase exponentially sharply with the number of trains and arrivaldeparture lines. Therefore, in order to reduce computation time, we take the trains' routes arrangement as tabu decision variable based on the scheme described in Table 4 . Then we get the near-optimal routing arrangement of each train (as shown in Figure 11) and the start time when trains enter the station (as shown in Table 7) based on the designed algorithm in a short time.

The routing arrangements can be seen clearly in Figure 11. From our intuitive, the objective that utilization balanced of the arrival-departure lines is not only satisfied but also meets the transfer relationship between $c_{1}$ and $c_{3}$. What is more, all trains are of punctuality. It is noteworthy that the results we obtained from the algorithm are the same as obtained directly from CPLEX solver, and the result suits the requirements of train operations absolutely. Therefore, it can be demonstrated that the proposed model can optimize the routes of trains and the algorithm can get a reasonable solution to ensure the punctuality of train and the rationality of routing.

5.2. Large-Scale Case Experiment. To test the effectiveness and efficiency of our proposed train routing problem model and approach, this section applies the proposed model to a real-world case study on the Jinan West high-speed railway station in China, which involves 17 arrival-departure lines including 4 main lines and 8 platforms to the operations of reception and departure. The network of railway is described by the method mentioned in Section 2.1 as shown in Figure 12.

In Figure 12, arrival-departure lines $I$ and $I I I$ are outbound main lines and $I I$ and $I V$ are inbound main lines, respective. Arrival-departure lines $I$ and $I I$ are used to receive and depart trains between Beijing and Shanghai, and the direction from Shanghai toward Beijing is defined as the inbound direction, while it is outbound direction. Similarly, arrival-departure lines $I I I$ and $I V$ are used to receiving and departing trains between Beijing and Qingdao, while the direction from Qingdao toward Beijing is defined as the inbound direction, whereas it is outbound direction. The 

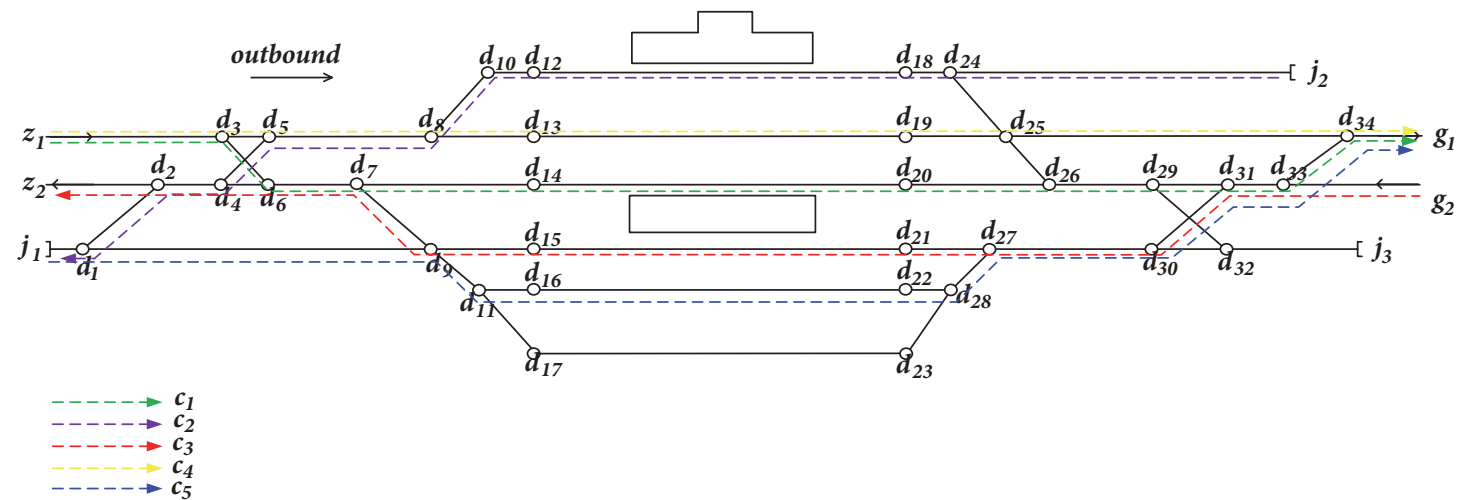

FIGURE 11: The route arrangements of the small case.

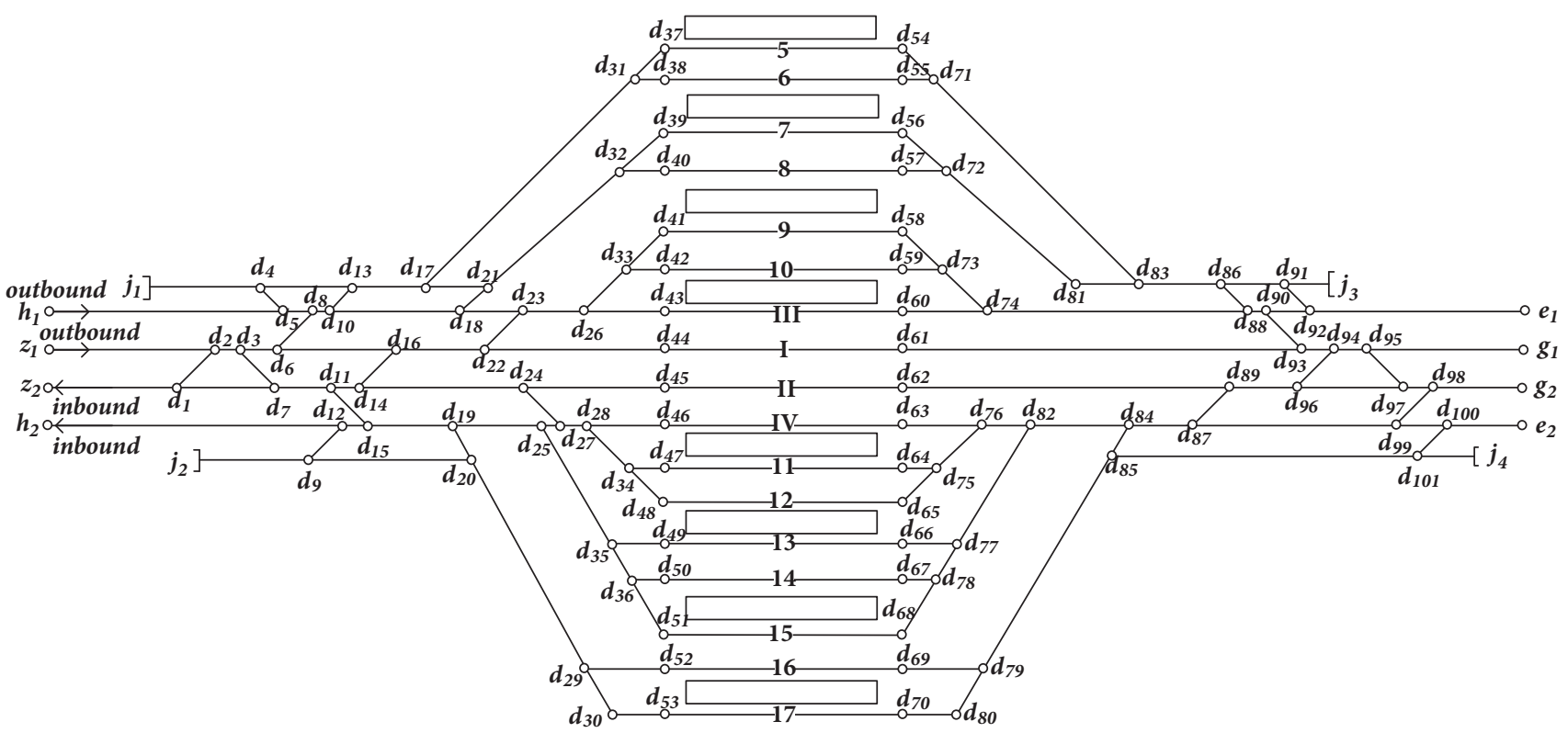

Figure 12: Construction of Jinan West Railway Station in China.

arrival-departure lines $5 \sim 10$ are located on the side of the outbound, while arrival-departure lines 11 17 are located on the side of the inbound. The distribution of the platform is shown in Figure 12. In addition, there are four locomotive waiting tracks $j_{1} \sim j_{4}$.

Owing to the scale of the station, the number of turnouts and crossovers is large, so it is difficult to collect the actual length between all track segments. Thus, the distances between various connection points are not listed here. It is described that if a train stops on the arrival-departure line which is far away from the main line, this would cost more travel time. In the real-world operation, trains need to align with the platform signs when they arrive at the station which need a little more time, and this need not be considered when trains depart from the station. In addition, in terms of our common sense, the trains' speed will decrease when arrive at the station due to the limit of lateral speed at turnouts. So there is insignificant difference in traveling time in the station between trains running at $300 \mathrm{~km} / \mathrm{h}$ and
$250 \mathrm{~km} / \mathrm{h}$. Combined with the calculation methods of track occupied time described in Section 2.4 and the large number of observation results in the control center, the occupancy time of each route is shown in Table 8 . The parameters $\alpha_{c}=1$ since the shunting operation is not regarded.

In the real-world station trains reception and departure operations, the nonstopped trains directly pass through the station on main lines, while the stopped trains can stop on any arrival-departure lines except main lines. However, if inbound trains enter the station from $g_{2}$ and stop on the arrival-departure line 5 in the station as shown in Figure 12, this not only causes route conflicts with the outbound trains but also brings inconvenience to the outbound trains, which occupies more equipment and increases the difficulty of operations. Therefore, the inbound train should occupy the arrival-departure line on the side of inbound main line correspondingly (i.e., inbound trains enter the station from $g_{2}$ or $e_{2}$ and stop at arrival-departure lines $\left.11 \sim 17\right)$. Like the outbound trains, namely, outbound trains enter the station 


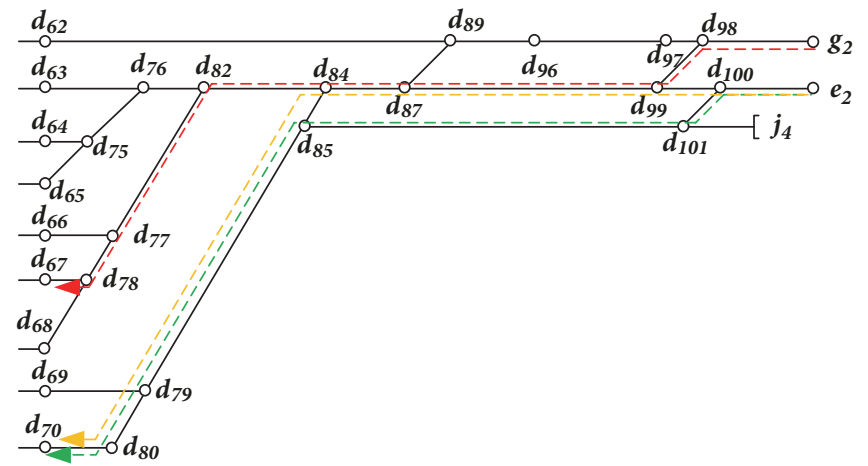

FIGURE 13: The parallel routes of two trains at bottleneck.

TABLE 8: Travel time of trains on the left bottleneck, the arrival-departure lines, and the right bottleneck.

\begin{tabular}{|c|c|c|c|c|c|}
\hline $\begin{array}{l}\text { Stopped } \\
\text { arrival-departure } \\
\text { line of outbound } \\
\text { trains }\end{array}$ & $\begin{array}{c}\text { Travel time of } \\
\text { reception at the } \\
\text { right bottleneck } \\
t_{c s}^{r}(\mathrm{~s})\end{array}$ & $\begin{array}{c}\text { Travel time of } \\
\text { departure at the left } \\
\text { bottleneck } \\
t_{c s}^{l e}(\mathrm{~s})\end{array}$ & $\begin{array}{c}\text { Stopped } \\
\text { arrival-departure line } \\
\text { of inbound trains }\end{array}$ & $\begin{array}{c}\text { Travel time of } \\
\text { reception at the left } \\
\text { bottleneck } \\
t_{c s}^{l}(\mathrm{~s})\end{array}$ & $\begin{array}{c}\text { Travel time of } \\
\text { departure at the right } \\
\text { bottleneck } \\
t_{c s}^{r e}(\mathrm{~s})\end{array}$ \\
\hline 11 & 100 & 80 & 5 & 125 & 105 \\
\hline 12 & 105 & 85 & 6 & 120 & 100 \\
\hline 13 & 110 & 90 & 7 & 115 & 95 \\
\hline 14 & 115 & 95 & 8 & 110 & 90 \\
\hline 15 & 120 & 100 & 9 & 105 & 85 \\
\hline 16 & 125 & 105 & 10 & 100 & 80 \\
\hline 17 & 130 & 110 & & & \\
\hline
\end{tabular}

from $z_{1}$ or $h_{1}$ and stop at arrival-departure lines 5 10. As a result, the trains' travel time on the left or right bottleneck is listed in Table 8. We select all trains passing through the Jinan West railway station between 16:00 and 19:00, that is, 46 trains (including 27 outbound trains and 19 inbound trains). The parameters are shown in Table 9.

Since only a period of time is selected, we assume that the railway stations are all vacant. The data of every track segment cannot be obtained accurately; thus the value of $t_{c_{1} c_{2}}^{\text {con }}$ is $1 \mathrm{~min}$. If the large instance is solved accurately, only the situation of a train occupying the arrival-departure lines needs to calculate $17^{46}$ times. Such a large scale problem belongs to the NP-hard as mentioned above. We adopt the heuristic algorithm designed, starting from a set of routes of 46 trains which is the best solution chosen through the iterations of method described in Table 3 to obtain the superior initial solution. Based on that, the tabu search algorithm is implied. We choose the routes with the lowest objective value as the target of the next search. Consequently the efficiency of searching a better solution is ensured. After that, the near-optimal solution with train routing is obtained within 254 seconds, and the results of different direction trains are shown in Tables 10 and 11, respectively (only a part of nodes clearly expresses the routes occupied by train duo to the large number of nodes).

As shown in Tables 10 and 11, it can be seen that the frequency of arrival-departure lines occupied are almost equal, which satisfies the objective of utilization balanced of them. The nonconflict parallels are occupied in the bottleneck as far as possible for two trains whose arrival time are close to each other. As for the inbound trains G134 and G4218, the arrival time of them are close and may cause a conflict in the right bottleneck. Therefore, routes are arranged on the parallel respectively as shown in Figure 13 (the red and green dotted lines represent the routes of the two trains, instead of the red and the yellow dotted line) to prevent the possibility of collision.

In addition, the planned start timetable to enter the station is obtained by the arrival time minus the travel time of the reception in the bottleneck according to Table 9, which is same with the actual start time calculated by the algorithm. That is, the model we propose and designed algorithm can get the trains routing arrangement in a high quality and short time based on the punctuality of trains. From the analysis results of the examples, the proposed model and the designed algorithm can solve the TRP efficiently.

It is worth mentioning that the problem we discussed is a NP-hard problem. Owing to the nonlinearity and numerous decision variables of the proposed mathematical model, there is neither proper commercial software available nor an algorithm which can solve such problems accurately at present. At the same time, the heuristic algorithm we designed can solve the problem accurately and efficiently as mentioned above. So we did not compare the designed algorithm with other methods in the large-scale case. 
TABLE 9: Parameters of trains passing through the Jinan West railway station.

\begin{tabular}{|c|c|c|c|c|c|c|c|}
\hline Train & Terminal & $\begin{array}{c}\text { Arrival } \\
\text { time }\end{array}$ & $\begin{array}{c}\text { Departure } \\
\text { time }\end{array}$ & $\begin{array}{l}\text { Stop time } \\
(\mathrm{min})\end{array}$ & Direction & $\begin{array}{c}\text { Origin/Destination in } \\
\text { station }\end{array}$ & Operations \\
\hline G30 & Beijing South & $16: 00$ & $16: 02$ & 2 & Inbound & $g_{2} / z_{2}$ & Passing \\
\hline G215 & Shanghai & $16: 05$ & $16: 08$ & 3 & Outbound & $z_{1} / g_{1}$ & Passing \\
\hline G191 & Qingdao & $16: 00$ & 16:09 & 9 & Outbound & $h_{1} / e_{1}$ & Passing \\
\hline G143 & Shanghai & $16: 10$ & $16: 12$ & 2 & Outbound & $z_{1} / g_{1}$ & Passing \\
\hline G168 & Beijing South & $16: 12$ & $16: 16$ & 4 & Inbound & $g_{2} / z_{2}$ & Passing \\
\hline G17 & Shanghai & $16: 22$ & $16: 24$ & 2 & Outbound & $z_{1} / g_{1}$ & Passing \\
\hline G132 & Beijing South & $16: 21$ & $16: 28$ & 7 & Inbound & $g_{2} / z_{2}$ & Passing \\
\hline G145 & Shanghai & $16: 15$ & $16: 34$ & 19 & Outbound & $z_{1} / g_{1}$ & Passing \\
\hline G322 & Beijing South & $16: 31$ & $16: 39$ & 8 & Inbound & $g_{2} / z_{2}$ & Passing \\
\hline G1203 & Shanghai & $16: 37$ & $16: 43$ & 6 & Outbound & $z_{1} / g_{1}$ & Passing \\
\hline G412 & Beijing South & $16: 37$ & $16: 44$ & 7 & Inbound & $g_{2} / z_{2}$ & Passing \\
\hline G193 & Qingdao & $16: 42$ & $16: 47$ & 5 & Outbound & $h_{1} / e_{1}$ & Passing \\
\hline G35 & Hangzhou East & $16: 51$ & $16: 53$ & 2 & Outbound & $z_{1} / g_{1}$ & Passing \\
\hline G56 & Beijing South & $16: 51$ & $16: 53$ & 2 & Inbound & $g_{2} / z_{2}$ & Passing \\
\hline G161 & Anqing & $16: 57$ & $16: 58$ & 2 & Outbound & $z_{1} / g_{1}$ & Passing \\
\hline G4 & Beijing South & $16: 59$ & $17: 01$ & 2 & Inbound & $g_{2} / z_{2}$ & Passing \\
\hline G53 & Hangzhou East & $17: 00$ & $17: 03$ & 3 & Outbound & $z_{1} / g_{1}$ & Passing \\
\hline G134 & Beijing South & $17: 09$ & $17: 11$ & 2 & Inbound & $g_{2} / z_{2}$ & Passing \\
\hline G4218 & Beijing South & $17: 01$ & $17: 15$ & 14 & Inbound & $e_{2} / h_{2}$ & Passing \\
\hline G351 & $\begin{array}{l}\text { Huangshan } \\
\text { North }\end{array}$ & $17: 17$ & $17: 19$ & 2 & Outbound & $z_{1} / g_{1}$ & Passing \\
\hline G45 & Jiangshan & $17: 22$ & $17: 24$ & 2 & Outbound & $z_{1} / g_{1}$ & Passing \\
\hline G475 & Rongcheng & $17: 29$ & $17: 31$ & 2 & Outbound & $h_{1} / e_{1}$ & Passing \\
\hline G194 & Beijing South & $17: 28$ & $17: 32$ & 4 & Inbound & $e_{2} / h_{2}$ & Passing \\
\hline G138 & Beijing South & $17: 33$ & $17: 36$ & 3 & Inbound & $g_{2} / z_{2}$ & Passing \\
\hline G1267 & Qingdao East & $17: 34$ & $17: 40$ & 6 & Outbound & $h_{1} / e_{1}$ & Passing \\
\hline G330 & Tianjin West & $17: 37$ & $17: 40$ & 3 & Inbound & $g_{2} / z_{2}$ & Passing \\
\hline $\mathrm{G} 21$ & Shanghai & $17: 39$ & $17: 41$ & 2 & Outbound & $z_{1} / g_{1}$ & Passing \\
\hline G147 & Shanghai & $17: 43$ & $17: 45$ & 2 & Outbound & $z_{1} / g_{1}$ & Passing \\
\hline G140 & Beijing South & $17: 41$ & $17: 45$ & 4 & Inbound & $g_{2} / z_{2}$ & Passing \\
\hline D6077 & Rongcheng & - & $17: 48$ & - & Outbound & $j_{1} / e_{1}$ & Originating \\
\hline G195 & Qingdao & $17: 57$ & 18:00 & 3 & Outbound & $h_{1} / e_{1}$ & Passing \\
\hline G18 & Beijing South & $17: 59$ & 18:01 & 2 & Inbound & $g_{2} / z_{2}$ & Passing \\
\hline G37 & Hangzhou East & $18: 02$ & 18:04 & 2 & Outbound & $z_{1} / g_{1}$ & Passing \\
\hline G164 & Beijing South & $17: 53$ & 18:06 & 13 & Inbound & $g_{2} / z_{2}$ & Passing \\
\hline G1235 & Shanghai & $18: 08$ & $18: 14$ & 6 & Outbound & $z_{1} / g_{1}$ & Passing \\
\hline G149 & Shanghai & $18: 13$ & $18: 18$ & 5 & Outbound & $z_{1} / g_{1}$ & Passing \\
\hline G142 & Beijing South & $18: 18$ & $18: 21$ & 3 & Inbound & $g_{2} / z_{2}$ & Passing \\
\hline G474 & Beijing South & $18: 27$ & $18: 29$ & 2 & Inbound & $e_{2} / h_{2}$ & Passing \\
\hline G1231 & Shanghai & $18: 17$ & $18: 29$ & 12 & Outbound & $z_{1} / g_{1}$ & Passing \\
\hline G197 & Qingdao & $18: 28$ & $18: 31$ & 3 & Outbound & $h_{1} / e_{1}$ & Passing \\
\hline G324 & Beijing South & $18: 25$ & $18: 33$ & 8 & Inbound & $g_{2} / z_{2}$ & Passing \\
\hline G39 & Hangzhou East & $18: 33$ & $18: 35$ & 2 & Outbound & $z_{1} / g_{1}$ & Passing \\
\hline G23 & Shanghai & $18: 39$ & $18: 41$ & 2 & Outbound & $z_{1} / g_{1}$ & Passing \\
\hline G52 & Tianjin West & $18: 46$ & $18: 49$ & 3 & Inbound & $g_{2} / z_{2}$ & Passing \\
\hline G1257 & Shanghai & $18: 50$ & $18: 53$ & 3 & Outbound & $z_{1} / g_{1}$ & Passing \\
\hline G153 & Shanghai & $18: 56$ & $18: 58$ & 2 & Outbound & $z_{1} / g_{1}$ & Passing \\
\hline
\end{tabular}

The "-" in the table indicates that the corresponding train is originating train. The entry time and stop time depend on the actual situation. It is assumed here that D6077 train enters from $j_{1}$ and departs after $30 \mathrm{~min}$. 
TABLE 10: The routes occupied by outbound trains through the Jinan West railway station between 16:00 and 19:00.

\begin{tabular}{|c|c|c|c|}
\hline Train & $\begin{array}{l}\text { Nodes of trains passing through at the left } \\
\text { bottleneck when arrive at the station. }\end{array}$ & $\begin{array}{l}\text { Arrival-departure } \\
\text { line }\end{array}$ & $\begin{array}{l}\text { Nodes of trains passing through at the right } \\
\text { bottleneck when depart from the station. }\end{array}$ \\
\hline G215 & $z_{1}, d_{6}, d_{8}, d_{26}, d_{41}$ & 9 & $d_{58}, d_{74}, d_{90}, d_{93}, g_{1}$ \\
\hline G191 & $h_{1}, d_{18}, d_{32}, d_{40}$ & 8 & $d_{57}, d_{72}, d_{81}, d_{86}, d_{88}, e_{1}$ \\
\hline G143 & $z_{1}, d_{6}, d_{8}, d_{26}, d_{41}$ & 9 & $d_{58}, d_{74}, d_{88}, d_{90}, d_{93}, g_{1}$ \\
\hline G17 & $z_{1}, d_{22}, d_{23}, d_{26}, d_{33}, d_{42}$ & 10 & $d_{59}, d_{73}, d_{74}, d_{90}, d_{93}, g_{1}$ \\
\hline G145 & $z_{1}, d_{6}, d_{8}, d_{10}, d_{13}, d_{17}, d_{37}$ & 5 & $d_{54}, d_{83}, d_{86}, d_{88}, d_{90}, d_{93}, g_{1}$ \\
\hline G1203 & $z_{1}, d_{22}, d_{23}, d_{26}, d_{33}, d_{42}$ & 10 & $d_{59}, d_{73}, d_{74}, d_{90}, d_{93}, g_{1}$ \\
\hline G193 & $h_{1}, d_{18}, d_{39}$ & 7 & $d_{56}, d_{81}, d_{91}, d_{92}, e_{1}$ \\
\hline G35 & $z_{1}, d_{22}, d_{23}, d_{26}, d_{41}$ & 9 & $d_{58}, d_{74}, d_{88}, d_{90}, d_{93}, g_{1}$ \\
\hline G161 & $z_{1}, d_{6}, d_{8}, d_{18}, d_{32}, d_{40}$ & 8 & $d_{57}, d_{72}, d_{81}, d_{86}, d_{88}, d_{90}, d_{93}, g_{1}$ \\
\hline G53 & $z_{1}, d_{6}, d_{8}, d_{26}, d_{41}$ & 9 & $d_{58}, d_{74}, d_{90}, d_{93}, g_{1}$ \\
\hline G351 & $z_{1}, d_{6}, d_{8}, d_{10}, d_{13}, d_{17}, d_{37}$ & 5 & $d_{54}, d_{83}, d_{86}, d_{88}, d_{90}, d_{93}, g_{1}$ \\
\hline G45 & $z_{1}, d_{22}, d_{23}, d_{26}, d_{33}, d_{42}$ & 10 & $d_{59}, d_{73}, d_{74}, d_{90}, d_{93}, g_{1}$ \\
\hline G475 & $h_{1}, d_{18}, d_{39}$ & 7 & $d_{56}, d_{81}, d_{91}, d_{92}, e_{1}$ \\
\hline G1267 & $h_{1}, d_{26}, d_{33}, d_{42}$ & 10 & $d_{59}, d_{73}, d_{74}, e_{1}$ \\
\hline G21 & $z_{1}, d_{6}, d_{8}, d_{18}, d_{32}, d_{39}$ & 7 & $d_{56}, d_{81}, d_{86}, d_{88}, d_{90}, d_{93}, g_{1}$ \\
\hline G147 & $z_{1}, d_{6}, d_{8}, d_{26}, d_{41}$ & 9 & $d_{58}, d_{74}, d_{90}, d_{93}, g_{1}$ \\
\hline D6077 & $j_{1}, d_{17}, d_{31}, d_{38}$ & 6 & $d_{35}, d_{71}, d_{83}, d_{86}, d_{88}, e_{1}$ \\
\hline G195 & $h_{1}, d_{26}, d_{33}, d_{42}$ & 10 & $d_{59}, d_{73}, d_{74}, e_{1}$ \\
\hline G37 & $z_{1}, d_{6}, d_{8}, d_{26}, d_{41}$ & 9 & $d_{58}, d_{74}, d_{90}, d_{93}, g_{1}$ \\
\hline G1235 & $z_{1}, d_{6}, d_{8}, d_{18}, d_{32}, d_{40}$ & 8 & $d_{57}, d_{72}, d_{81}, d_{86}, d_{88}, d_{90}, d_{93}, g_{1}$ \\
\hline G149 & $z_{1}, d_{6}, d_{8}, d_{10}, d_{13}, d_{17}, d_{31}, d_{38}$ & 6 & $d_{55}, d_{71}, d_{83}, d_{86}, d_{88}, d_{90}, d_{93}, g_{1}$ \\
\hline G1231 & $z_{1}, d_{6}, d_{8}, d_{18}, d_{32}, d_{39}$ & 7 & $d_{56}, d_{81}, d_{86}, d_{88}, d_{90}, d_{93}, g_{1}$ \\
\hline G197 & $h_{1}, d_{18}, d_{32}, d_{40}$ & 8 & $d_{57}, d_{72}, d_{81}, d_{86}, d_{88}, e_{1}$ \\
\hline G39 & $z_{1}, d_{6}, d_{8}, d_{26}, d_{41}$ & 9 & $d_{58}, d_{74}, d_{90}, d_{93}, g_{1}$ \\
\hline G23 & $z_{1}, d_{22}, d_{23}, d_{26}, d_{33}, d_{42}$ & 10 & $d_{59}, d_{73}, d_{74}, d_{90}, d_{93}, g_{1}$ \\
\hline G1257 & $z_{1}, d_{6}, d_{8}, d_{26}, d_{41}$ & 9 & $d_{58}, d_{74}, d_{90}, d_{93}, g_{1}$ \\
\hline G153 & $z_{1}, d_{6}, d_{8}, d_{18}, d_{32}, d_{40}$ & 8 & $d_{57}, d_{72}, d_{81}, d_{86}, d_{88}, d_{90}, d_{93}, g_{1}$ \\
\hline
\end{tabular}

\section{Conclusions}

This paper focuses on modeling and solving the TRP based on considering heterogeneous trains and detailed structure of the rail station. The main research work and conclusions are summarized as follows:

(1) A detailed mathematical formulation for TRP describes the routing arrangement in a large and complex high-speed railway station. The turnout node and the arrival-departure line node are defined to describe the layout of the railway station instead of the traditional railway network. The heterogeneous trains are taken into account; and the potential collisions of trains and convenience for passengers transferring at station are considered as constraints. Then we propose a method to calculate the occupied time of each track and describe the TRP problem more completely and realistically.

(2) A high-efficient algorithm based on tabu search is proposed based on the proposed model. We set two different neighborhoods for searching incumbent solution and tabu move to search the near-optimal solution. The scale of first neighborhood is smaller to search in a short time, and the second neighborhood is wilder in order to avoid empty neighborhoods as far as possible. Then we set two strategies of them, respectively.

(3) The correctness of the proposed model is verified with a small example that includes all types of trains and operations. The results obtained by the proposed tabu search algorithm are the same as those obtained directly from CPLEX solver. The result is fully in line with the requirements of train operations. In the large scale case, we chose the actual trains within 3 hours passing through the Jinan West railway station which involves 17 arrival-departure lines. The excellent solutions are obtained within 254 seconds by the designed algorithm.

It is realistic and easy to understand our proposed model. However, it has the characteristics of non-linearity and has 
TABLE 11: The routes occupied by inbound trains through the Jinan West railway station between 16:00 and 19:00.

\begin{tabular}{|c|c|c|c|}
\hline Train & $\begin{array}{l}\text { Nodes of trains passing through at the right } \\
\text { bottleneck when arrive at the station. }\end{array}$ & $\begin{array}{l}\text { Arrival-departure } \\
\text { line }\end{array}$ & $\begin{array}{l}\text { Nodes of trains passing through at the left } \\
\text { bottleneck when depart from the station. }\end{array}$ \\
\hline G30 & $g_{2}, d_{98}, d_{99}, d_{76}, d_{75}, d_{64}$ & 11 & $d_{47}, d_{34}, d_{28}, d_{27}, d_{24}, z_{2}$ \\
\hline G168 & $g_{2}, d_{89}, d_{87}, d_{76}, d_{65}$ & 12 & $d_{48}, d_{28}, d_{15}, d_{11}, z_{2}$ \\
\hline G132 & $g_{2}, d_{98}, d_{99}, d_{82}, d_{78}, d_{67}$ & 14 & $d_{50}, d_{36}, d_{25}, d_{15}, d_{11}, z_{2}$ \\
\hline G322 & $g_{2}, d_{98}, d_{99}, d_{76}, d_{75}, d_{64}$ & 11 & $d_{47}, d_{34}, d_{28}, d_{27}, d_{24}, z_{2}$ \\
\hline G412 & $g_{2}, d_{98}, d_{99}, d_{82}, d_{77}, d_{66}$ & 13 & $d_{49}, d_{35}, d_{25}, d_{15}, d_{11}, z_{2}$ \\
\hline G56 & $g_{2}, d_{89}, d_{87}, d_{82}, d_{68}$ & 15 & $d_{51}, d_{25}, d_{15}, d_{11}, z_{2}$ \\
\hline G4 & $g_{2}, d_{98}, d_{99}, d_{82}, d_{77}, d_{66}$ & 13 & $d_{49}, d_{35}, d_{25}, d_{15}, d_{11}, z_{2}$ \\
\hline G134 & $g_{2}, d_{98}, d_{99}, d_{82}, d_{78}, d_{67}$ & 14 & $d_{50}, d_{36}, d_{25}, d_{15}, d_{11}, z_{2}$ \\
\hline G4218 & $e_{2}, d_{100}, d_{101}, d_{85}, d_{80}, d_{70}$ & 17 & $d_{53}, d_{30}, d_{19}, h_{2}$ \\
\hline G194 & $e_{2}, d_{76}, d_{75}, d_{64}$ & 11 & $d_{47}, d_{34}, d_{28}, h_{2}$ \\
\hline G138 & $g_{2}, d_{89}, d_{87}, d_{84}, d_{79}, d_{69}$ & 16 & $d_{52}, d_{29}, d_{19}, d_{15}, d_{11}, z_{2}$ \\
\hline G330 & $g_{2}, d_{98}, d_{99}, d_{82}, d_{77}, d_{66}$ & 13 & $d_{49}, d_{35}, d_{25}, d_{15}, d_{11}, z_{2}$ \\
\hline G140 & $g_{2}, d_{98}, d_{99}, d_{82}, d_{78}, d_{67}$ & 14 & $d_{50}, d_{36}, d_{25}, d_{15}, d_{11}, z_{2}$ \\
\hline G18 & $g_{2}, d_{89}, d_{87}, d_{76}, d_{65}$ & 12 & $d_{48}, d_{28}, d_{15}, d_{11}, z_{2}$ \\
\hline G164 & $g_{2}, d_{89}, d_{87}, d_{82}, d_{68}$ & 15 & $d_{51}, d_{25}, d_{15}, d_{11}, z_{2}$ \\
\hline G142 & $g_{2}, d_{98}, d_{99}, d_{82}, d_{77}, d_{66}$ & 13 & $d_{49}, d_{35}, d_{25}, d_{15}, d_{11}, z_{2}$ \\
\hline G474 & $e_{2}, d_{76}, d_{65}$ & 12 & $d_{48}, d_{28}, h_{2}$ \\
\hline G324 & $g_{2}, d_{89}, d_{87}, d_{84}, d_{79}, d_{69}$ & 16 & $d_{52}, d_{29}, d_{19}, d_{15}, d_{11}, z_{2}$ \\
\hline G52 & $g_{2}, d_{89}, d_{87}, d_{76}, d_{65}$ & 12 & $d_{48}, d_{28}, d_{15}, d_{11}, z_{2}$ \\
\hline
\end{tabular}

difficulties to solve the mathematical formulas. Therefore, one possible future direction is to improve the model. Second, the tabu search algorithm needs to set effective rules to generate the incumbent solution's neighborhood and rules for tabu move. In this respect, further improvements are needed to obtain a better near-optimal solution more efficiently. More importantly, future research does not restrict to a railway stations. With the rapid development of high-speed railway, there are more train routing and scheduling problems, train rerouting and rescheduling optimization problems, etc., which need to be further studied.

\section{Data Availability}

Previously reported [construction and related data of Jinan West Railway Station in China] data were used to support this study and are available at [https://www.researchgate.net/ publication/276080928_Using_Simulated_Annealing_in_a_ Bottleneck_Optimization_Model_at_Railway_Stations].

These prior studies (and datasets) are cited at relevant places within the text as [20] (i.e., [2]). The [trains information passing through the Jinan West railway station] data used to support the findings of this study have been deposited in the [Railway Customer Service Center of China] repository ([https://kyfw.12306.cn/otn/leftTicket/init]).

\section{Conflicts of Interest}

The authors declare that there are no conflicts of interest regarding the publication of this paper.

\section{Acknowledgments}

This research was supported by the Research Foundation of State Key Laboratory of Rail Traffic Control and Safety, Beijing Jiaotong University, China (nos. RCS2017ZT012, RCS2018ZZ001, and RCS2018ZZ003), and the Fundamental Research Funds for the Central Universities (no. 2018YJS193).

\section{References}

[1] A. Caprara, L. Galli, and P. Toth, "Solution of the train platforming problem," Transportation Science, vol. 45, no. 2, pp. 246-257, 2011.

[2] L. J. Kang, J. J. Wu, and H. J. Sun, "Using simulated annealing in a bottleneck optimization model at railway stations," Journal of Transportation Engineering, vol. 138, no. 11, pp. 1396-1402, 2012.

[3] A. D'Ariano, D. Pacciarelli, and M. Pranzo, "Assessment of flexible timetables in real-time traffic management of a railway bottleneck," Transportation Research Part C: Emerging Technologies, vol. 16, no. 2, pp. 232-245, 2008.

[4] W. Z. Jia, B. H. Mao, T. K. Ho, H. D. Liu, and B. Yang, "Bottleneck detection of track allocation schemes at rail station by Petri nets," Journal of Transportation Systems Engineering \& Information Technology, vol. 9, no. 6, pp. 136-141, 2009.

[5] A. Billionnet, "Using integer programming to solve the trainplatforming problem," Transportation Science, vol. 37, no. 2, pp. 213-222, 2003.

[6] R. Qiao, X. Zhu, and W. Yan, "Optimized utilization of station arrival and departure tracks for passenger dedicated lines," Advanced Materials Research, vol. 468-471, pp. 2450-2454, 2012.

[7] P. J. Zwaneveld, L. G. Kroon, and S. P. M. Van Hoesel, "Routing trains through a railway station based on a node packing 
model," European Journal of Operational Research, vol. 128, no. 1, pp. 14-33, 2001.

[8] F. Corman, A. D’Ariano, D. Pacciarelli, and M. Pranzo, "A tabu search algorithm for rerouting trains during rail operations," Transportation Research Part B: Methodological, vol. 44, no. 1, pp. 175-192, 2010.

[9] V. Cacchiani and P. Toth, "Nominal and robust train timetabling problems," European Journal of Operational Research, vol. 219, no. 3, pp. 727-737, 2012.

[10] W. Zhou and H. Teng, "Simultaneous passenger train routing and timetabling using an efficient train-based Lagrangian relaxation decomposition," Transportation Research Part B: Methodological, vol. 94, pp. 409-439, 2016.

[11] Y. Xu, B. Jia, A. Ghiasi, and X. Li, "Train routing and timetabling problem for heterogeneous train traffic with switchable scheduling rules," Transportation Research Part C: Emerging Technologies, vol. 84, pp. 196-218, 2017.

[12] F. Li, Z. Gao, K. Li, and D. Z. W. Wang, "Train routing model and algorithm combined with train scheduling," Journal of Transportation Engineering, vol. 139, no. 1, pp. 81-91, 2013.

[13] W. Liu, X. Zhu, and L. Kang, "Real-Time Track Reallocation for Emergency Incidents at Large Railway Stations," Mathematical Problems in Engineering, vol. 2015, Article ID 296394, 11 pages, 2015.

[14] X. Zhou and M. Zhong, "Bicriteria train scheduling for highspeed passenger railroad planning applications," European Journal of Operational Research, vol. 167, no. 3, pp. 752-771, 2005.

[15] W. Fang, S. Yang, and X. Yao, "A Survey on Problem Models and Solution Approaches to Rescheduling in Railway Networks," IEEE Transactions on Intelligent Transportation Systems, vol. 16, no. 6, pp. 2997-3016, 2015.

[16] L. G. Kroon, H. E. Romeijn, and P. J. Zwaneveld, "Routing trains through railway stations: complexity issues," European Journal of Operational Research, vol. 98, no. 3, pp. 485-498, 1997.

[17] F. Corman and L. Meng, "A review of online dynamic models and algorithms for railway traffic control," IEEE Transactions on Intelligent Transportation Systems, vol. 16, no. 3, pp. 1274-1284, 2015.

[18] R. Ahuja, C. Cunha, and G. Sahin, "Network models in railroad planning and scheduling," in Tutorials in Operations Research, H. Greenberg and J. Smith, Eds., vol. 1, pp. 54-101, 2005.

[19] M. Carey and I. Crawford, "Scheduling trains on a network of busy complex stations," Transportation Research Part B: Methodological, vol. 41, no. 2, pp. 159-178, 2007.

[20] B. He, R. Song, S. He, and Y. Xu, "High-Speed Rail Train Timetabling Problem: A Time-Space Network Based Method with an Improved Branch-and-Price Algorithm," Mathematical Problems in Engineering, vol. 2014, Article ID 641562, 15 pages, 2014.

[21] J. Qi, L. Yang, Y. Gao, S. Li, and Z. Gao, "Integrated multitrack station layout design and train scheduling models on railway corridors," Transportation Research Part C: Emerging Technologies, vol. 69, pp. 91-119, 2016.

[22] M. Samà, A. D’Ariano, F. Corman, and D. Pacciarelli, "A variable neighbourhood search for fast train scheduling and routing during disturbed railway traffic situations," Computers \& Operations Research, vol. 78, pp. 480-499, 2017.

[23] G. L. Giacco, A. D’Ariano, and D. Pacciarelli, "Rolling stock rostering optimization under maintenance constraints," Journal of Intelligent Transportation Systems: Technology, Planning, and Operations, vol. 18, no. 1, pp. 95-105, 2014.
[24] F. Glover, "Future paths for integer programming and links to artificial intelligence," Computers \& Operations Research, vol. 13, no. 5, pp. 533-549, 1986. 


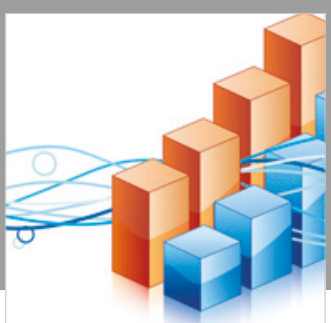

Advances in

Operations Research

\section{-n-m}
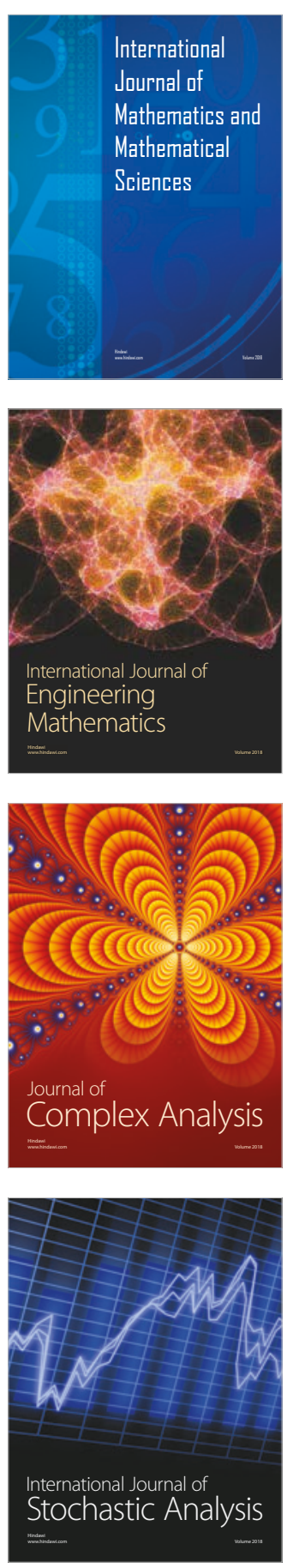
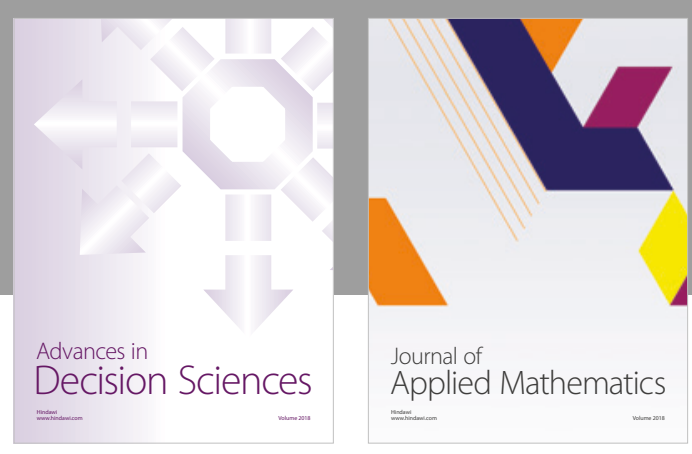

Journal of

Applied Mathematics
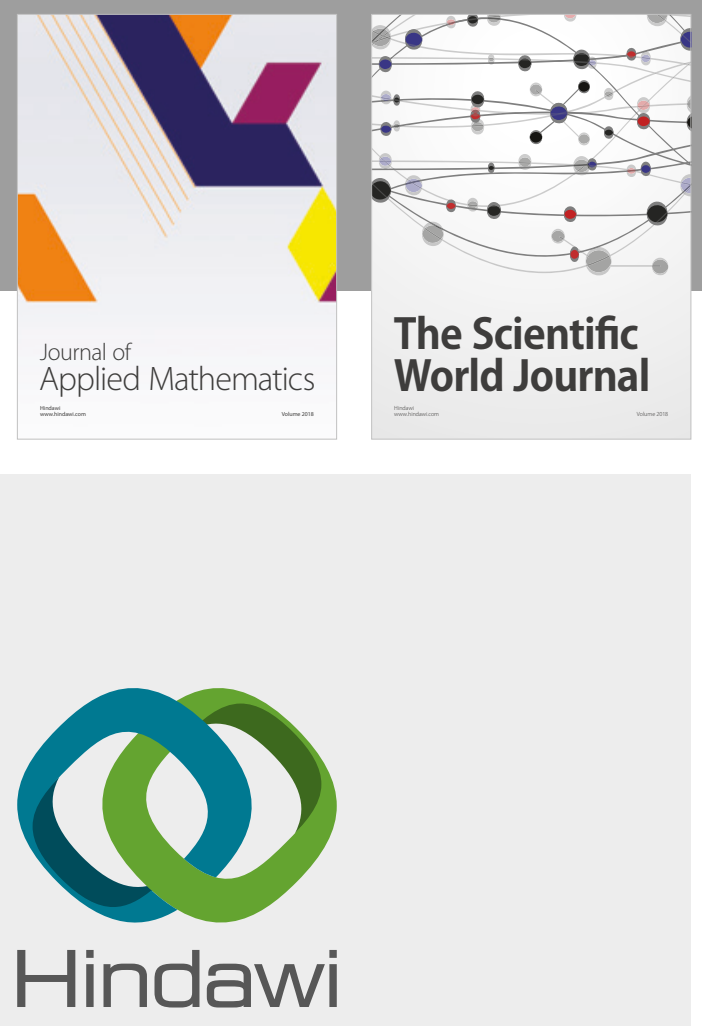

Submit your manuscripts at

www.hindawi.com

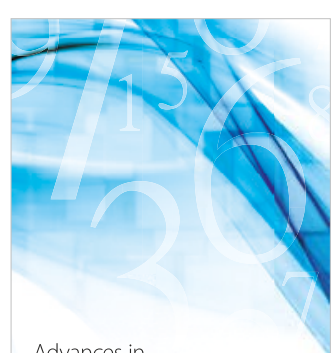

Advances in
Numerical Analysis
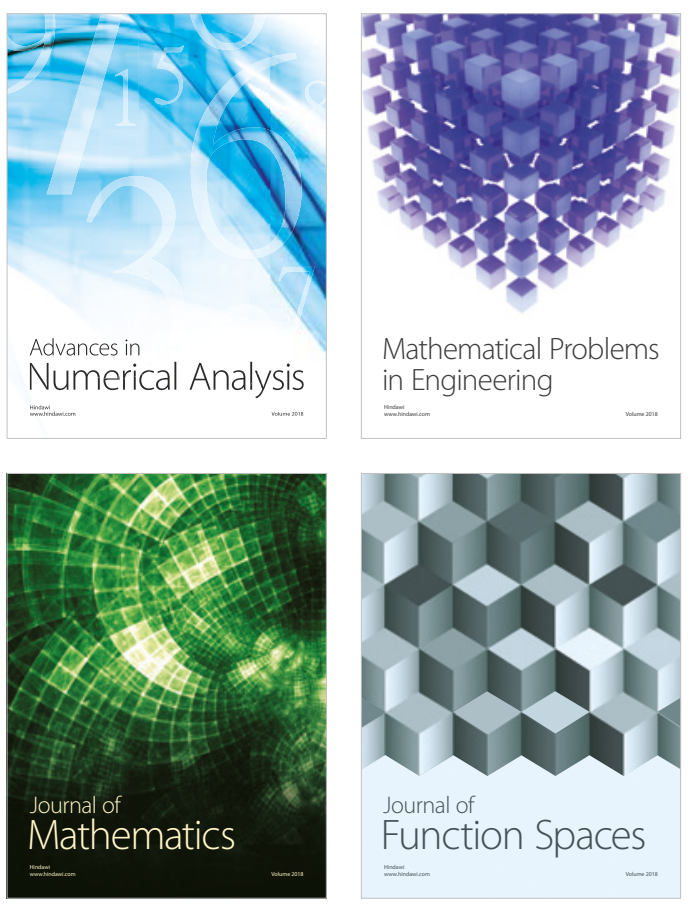

Mathematical Problems in Engineering

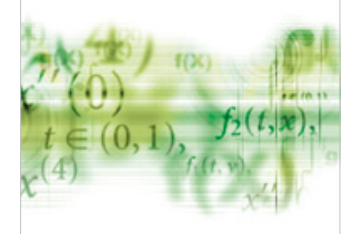

International Journal of

Differential Equations

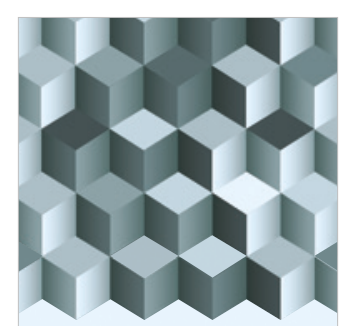

Journal of

Function Spaces

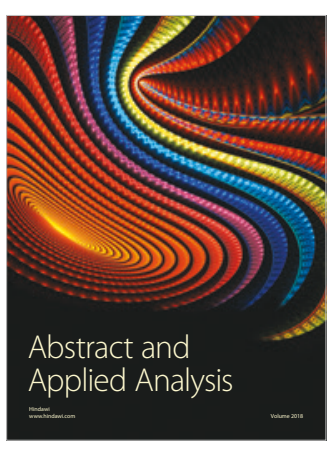

The Scientific

World Journal

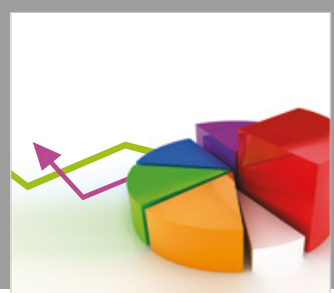

Journal of

Probability and Statistics
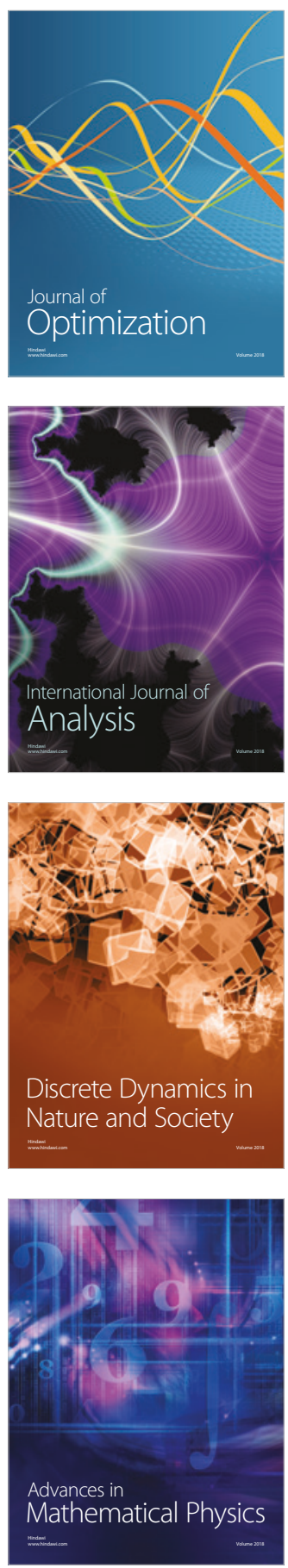